\title{
1 Peripheral microtubules ensure asymmetric furrow positioning in neural stem cells
}

2

3 Running title: Peripheral microtubules not the spindle midzone, position the asymmetric

4 division furrow in neural stem cells.

6 Alexandre Thomas ${ }^{1}$, Emmanuel Gallaud ${ }^{1}$, Aude Pascal $^{1}$, Laurence Serre ${ }^{2}$, Isabelle Arnal ${ }^{2}$, 7 Laurent Richard-Parpaillon ${ }^{1}$, Matthew Scott Savoian $^{3}$ and Régis Giet ${ }^{1 \#}$.

1. Univ Rennes, CNRS, IGDR (Institut de Génétique et Développement de Rennes) - UMR 6290, F-35000 Rennes, France

2. Univ. Grenoble Alpes, Inserm U1216, CEA, CNRS, Grenoble Institut Neurosciences, GIN, 38000 Grenoble, France.

3. Massey University, School of Fundamental Sciences, 4410, Palmerston North, New Zealand

\#. Corresponding author: regis.giet@univ-rennes 1.fr

\section{Abstract}

Neuroblast (NB) cell division is characterized by a basal positioning of the cleavage furrow resulting in a large difference in size between the future daughter cells. In animal cells, furrow placement and assembly is governed by centralspindlin, a highly conserved complex that accumulates at the equatorial cell cortex of the future cleavage site and at the spindle midzone. In contrast to model systems studied so far, these two centralspindlin populations are spatially and temporally separated in NBs. A cortical leading pool is located at the basal cleavage furrow site and a second pool accumulates at the midzone before travelling to the site of the basal cleavage furrow during cytokinesis completion. By manipulating microtubule (MT) dynamics, we show that the cortical centralspindlin population requires peripheral astral microtubules and the Chromosome Passenger Complex (CPC) for efficient recruitment. Loss of this pool does not prevent cytokinesis but enhances centralspindlin levels at the midzone leading to furrow repositioning towards the equator and decreased size asymmetry between daughter cells. Together these data reveal that the asymmetrical furrow placement characteristic of NBs results from a competition between spatially and functionally separate centralspindlin pools in which the cortical pool is dominant and requires peripheral astral microtubules. 


\section{Introduction}

Cytokinesis in somatic cells ensures the equal partitioning of the segregated chromosomes and is responsible for the division of the mother cell's cytoplasm into two daughters. This process requires the highly orchestrated assembly and constriction of an acto-myosin contractile ring, usually at the cell's center. The use of various model systems has clearly established that the mitotic spindle defines the position of the contractile ring and the resulting cleavage furrow ${ }^{1,2}$. Two populations of mitotic spindle microtubules (MTs) have been shown to trigger the assembly of the contractile machinery during late anaphase. The first is a subpopulation of astral MTs. These MTs emanate from the centrosomes to the equatorial cortex where they deliver furrow-inducing signals ${ }^{3-5}$. The second population, comprises the spindle midzone, a region of antiparallel MT overlap and interdigitation within the central spindle that assembles between the decondensing daughter nuclei. In many symmetrically dividing somatic cell types the relative contribution of these two populations has been difficult to unambiguously determine due to their close proximity at the cell's equator. Yet, experiments during the last few decades have helped to propose a common mechanism across model systems in which the furrow-inducing signals emanate from both cortical proximal astral MTs and the spindle midzone with each acting in parallel. However, these pathways do not appear to be equivalent. For instance, if the furrow is located away from the midzone, it will regress and a new one will be established at the midzone location 1,3,6,7. Thus, in equatorially dividing cells, the spindle midzone pathway acts dominantly and can reset furrowing.

Centralspindlin is the main orchestrator of furrowing. This protein complex is a tetramer composed of two subunits of the Kinesin 6 (Pavarotti-klp in Drosophila melanogaster) and two subunits of the MgcRacGAP (Tumbleweed in Drosophila melanogaster). Tumbleweed is essential for the activation of the Rho-GEF Ect2 (Pebble in Drosophila melanogaster). The formation of Rho-GTP triggers the local activation of Rho Kinase and phosphorylation of non-muscle Myosin Regulatory Light Chain, an event that stimulates myosin activation and ultimately drives cytoplasmic cleavage ${ }^{8,9}$. While it is well established that centralspindlin acts along central spindle MTs and accumulates at the cell cortex equator to promote symmetrical cleavage, far less is known about how this complex governs asymmetrical divisions. Drosophila neural stem cells (Neuroblasts, NBs) are characterized by a biased furrow placement towards the basal region of the cell. Asymmetric cytokinesis triggers the formation of a large apically positioned cell that retains the NB identity, and a small basal ganglion mother cell (GMC) that will undergo differentiation ${ }^{10}$. Asymmetry is apparent prior 
71 to furrow initiation and can be detected during early anaphase as myosin redistributes from 72 around the cortex to a more basal position ${ }^{11}$. Previous studies have shown that this process is 73 under the strict control of the NB polarity machinery but is also influenced by the spindle 74 midzone and the Chromosome Passenger Complex (CPC) ${ }^{12,13}$. To better understand the 75 mechanism of furrow positioning in asymmetrical cytokinesis, we have genetically 76 manipulated spindle size and MTs dynamics in Drosophila NBs. Our data indicate that the 77 mechanisms dictating asymmetrical daughter cell size are extremely robust and tolerate 78 increases in spindle length and shape. We report that furrowing initiates away from the 79 midzone, in a basal position through the action of a subcortical centralspindlin pool targeted 80 by peripheral astral microtubules. When these MTs are ablated, centralspindlin recruitment at 81 the furrow is impaired and becomes abnormally enriched at the midzone causing 82 repositioning of the cleavage site, thus affecting the size asymmetry of the daughter cells. 83 Together these results reveal that unlike most systems, in Drosophila NBs that are 84 characterized by a high level of cell size asymmetry during cell division, a population of 85 peripheral astral MTs, and not the spindle midzone, defines and maintains asymmetric 86 cleavage furrow positioning. 


\section{Molecular biology}

Msps cDNA was provided by G. Rogers (University of Arizona, USA), amplified by PCR and inserted into pENTR (Life Technologies) to generate the pENTR-Msps entry clone. pENTR-Ensc has been previously described ${ }^{14}$. The pENTR-Feo and the pENTR-Tum entry clones were obtained from P.P D'Avino (University of Cambridge, UK). The pENTR-Feo and pENTR-Msps entry clones were each subsequently recombined into pTWV and pTWR (Carnegie Institute, USA) to generate constructs allowing the expression of Feo-VenusFP and Msps-RFP fusion proteins, respectively, under the control of the GAL4 protein using the Gateway recombination cloning technology (Life Technologies). pENTR-Ensc was recombined into pDEST-MBP (a gift from H. Ohkura, University of Edinburgh, UK) to allow the expression of a recombinant Ensconsin protein with a C-terminal MBP tag.

\section{Fly stocks}

103 All flies were maintained under standard conditions at $25^{\circ} \mathrm{C}$. The ensconsin mutant fly stocks ensc $\Delta$ null and ensc $\Delta N$, referred to ensc flies, were characterized previously (Sung et al 2008). UAS-Ensconsin-Venus transgenic flies, overexpressing Ensconsin-Venus, have been described ${ }^{15}$. UAS-Feo-Venus and UAS-Msps-RFP overexpressing flies were obtained from BestGene (USA) following P-element mediated transformation. UAS-Klp10A flies were supplied by C. Dahmann (Max Planck Institute, Germany) ${ }^{16}$. UAS-Klp67A (ID \# F001232) stock was obtained from FlyORF ${ }^{17}$. UAS-Klp67A-RNAi (VDRC ID 52105) and UAS-Mad2-

110 RNAi (VDRC ID 106003) transgenic fly lines were obtained from the Vienna Drosophila 111 RNAi Center ${ }^{18}$. Sqh-GFP ${ }^{19}$, UAS-GFP-Pav-klp and Ubiquitin- $\beta$ tub-GFP expressing flies ${ }^{20,}$

$112{ }^{21}$ were supplied by R. Karess (Institut Jacques Monod, France) and by D. Glover (University 113 of Cambridge, UK), respectively. The Pavarotti mutant pav ${ }^{B 200}$ flies were obtained from E.

114 Montembault (Institut Europeen de Chimie et Biologie, France) ${ }^{22}$ and Survivin mutant allele $115 s v n^{2180}$ flies were courtesy of Jean-René Hyunh (College de France, France) ${ }^{23}$. Flies 116 expressing the membrane-localized PH-PLC $\delta$-GFP and PH-PLC $\delta$-RFP proteins were

117 provided by A. Guichet (Institut Jacques Monod, France) ${ }^{24,25}$. RFP-Tubulin flies were 118 provided by R. Basto (Institut Currie, France). The GFP-AurA expressing fly stock was 119 described previously ${ }^{26}$. The following stocks were obtained from the Bloomington Stock 120 Center: Feo-GFP expressed under the ubiquitin promoter (BDSC 59273, ${ }^{27}$ ), sas $-4^{\text {s2214 }}$ mutant 
121 (BDSC 12119, ${ }^{28}$ ), 69B-Gal4 (BDSC 1774), Insc-Gal4 (BDSC 8751), UAS-mCherry- $\alpha$ -

122 tubulin (BDSC 25774 and BDSC 25773). The 69B-Gal4 fly stock was used to drive over-

123 expression in the fly CNS for the following UAS regulated transgenes: Ensconsin, Klp67A,

124 Msps, GFP-Pav-Klp together with Mad2 RNAi and UAS-mCherry. The Insc-Gal4 strain was

125 used to drive over-expression of Ensconsin, Klp10A, UAS- GFP-Pav-klp, Feo-Venus and

126 mCherry- $\alpha$ tubulin transgenes in the central brain.

127

128 Production of recombinant proteins

$129 \mathrm{MBP}$ and Ensconsin-MBP were induced in E.coli, for $4 \mathrm{~h}$ at $25^{\circ} \mathrm{C}$. The proteins were purified

130 on amylose column as described by the manufacturer (BioLabs) and stored in small aliquots

131 at $-80^{\circ} \mathrm{C}$.

132

133 TIRF microscopy and analysis of MT dynamics

134 Tubulin was purified from bovine brain and fluorescently labeled with ATTO 488 and ATTO

135565 or biotinylated as described before ${ }^{29,30}$. Briefly, microtubule seeds were prepared from

136 biotinylated and ATTO-565-labeled tubulin in the presence of Guanosine-5'-[( $\alpha, \beta)$ -

137 methyleno]triphosphate (GMPCPP) in BRB80 buffer (80 mM Pipes, $1 \mathrm{mM}$ EGTA, $1 \mathrm{mM}$

$138 \mathrm{MgCl}_{2}, \mathrm{pH}$ 6.74) ${ }^{30}$. Flow chambers were prepared with functionalized silane-PEG-biotin

139 coverslips and silane-PEG glass slides, as previously described (Ramirez-Rios et al., 2017).

140 The chamber was successively perfused at room temperature with neutravidin $(25 \mu \mathrm{g} / \mathrm{ml}$ in

141 1\% BSA in BRB80), PLL-g-PEG (2 kD, $0.1 \mathrm{mg} / \mathrm{ml}$ in $10 \mathrm{mM}$ Hepes, pH 7.4), BSA (1\% in

142 BRB80 buffer) and microtubule seeds. The following assembly mixture was then injected: 14

$143 \mu \mathrm{M}$ tubulin (containing $15 \%$ ATTO-488-labeled tubulin) without or with $200 \mathrm{nM}$ MBP or

144 MBP-Ensconsin in TIRF assay buffer (4 nM DTT, $50 \mathrm{mM} \mathrm{KCl}, 1 \% \mathrm{BSA}, 1 \mathrm{mg} / \mathrm{mL}$ glucose,

$14570 \mu \mathrm{g} / \mathrm{mL}$ catalase, $580 \mu \mathrm{g} / \mathrm{mL}$ glucose oxidase, $0.05 \%$ methylcellulose (4000 centipoise) in

146 BRB80). Time-lapse images were recorded at $35^{\circ} \mathrm{C}$ at a rate of one frame per 5 seconds on an

147 inverted Eclipse Ti Nikon microscope equipped with an Apochromat 60X1.49 N.A oil

148 immersion objective, an iLas ${ }^{2}$ TIRF system (Roper Scientific), and a cooled charge-coupled

149 device camera (EMCCD Evolve 512, Photometrics) controlled by MetaMorph 7.7.5 software.

150 Microtubule dynamic parameters were analyzed in Image $\mathbf{J}$ on kymographs obtained using an

151 in-house KymoTool macro (available upon request to eric.denarier@univ-grenoble-alpes.fr).

152 Growth and shrinkage rates were determined from the slopes of microtubule growth and

153 shrinkage phases. The catastrophe and rescue frequencies were calculated by dividing the 
154 number of events per microtubule by the time spent in growing and shrinking states, 155 respectively.

156

157 Antibodies and Western blotting

158 The following antibodies and concentrations were used in this study: polyclonal rabbit anti159 Msps (1:5000) provided by J. Raff ${ }^{31}$, polyclonal rabbit anti-Klp67A (1:500) supplied by G.

160 Goshima ${ }^{32}$, polyclonal rabbit anti-Klp10A (1:1000) was courtesy of G. Rogers ${ }^{33}$ and rabbit

161 anti-Myosin (1:2000) was provided by R. Karess ${ }^{34}$. The anti-Ensconsin antibody raised 162 against the Kinesin binding domain has been previously described ${ }^{14}$. Rabbit anti-PKC $\zeta$ (C-20, 163 1:200) and anti-actin polyclonal antibodies (sc-1616, 1:5000) were obtained from Santa Cruz 164 Technology. Monoclonal mouse anti-alpha Tubulin (clone DM1A, T2199; 1:500) and rabbit 165 polyclonal anti-phosphorylated histone H3 (Ser10) (06570, 1:500) antibodies were obtained 166 from Millipore. Monoclonal rat anti-Miranda antibody (ab197788, 1:1000) was obtained from 167 Abcam. Secondary antibodies were labelled with either Alexa Fluor-conjugated (1:1000) or 168 peroxidase-conjugated secondary antibodies (1:5000), each obtained from Life Technologies. 169 For Western Blotting ECL reagents were purchased from ThermoFisher.

\section{Live cell microscopy}

172 Third-instar larval brains were dissected in Schneider's Drosophila medium supplemented 173 with $10 \%$ FCS. Isolated brains were loaded and mounted on stainless steel slides, and the 174 preparations were sealed with mineral oil (Sigma-Aldrich) as previously described ${ }^{14}$. For MT 175 depolymerization experiments, larval brains were incubated during $30 \mathrm{~min}$ in the above 176 medium supplemented with colchicine at a final concentration of $15 \mu \mathrm{M}$. After incubation, 177 brains were mounted and processed for live cell imaging.

178 Images were acquired at $25^{\circ} \mathrm{C}$ using a CSU-X1 spinning-disk system mounted on an inverted 179 microscope (Elipse Ti; Nikon) equipped with a 60X 1.4 NA objective. At 20, 30 or $60 \mathrm{sec}$ 180 intervals $10 \mathrm{z}$-steps were acquired with $1 \mu \mathrm{m}$ intervals. Fluorescent protein probes were 181 excited with $488 \mathrm{~nm}$ or $561 \mathrm{~nm}$ laser light and the images were captured using a sCMOS 182 ORCA-Flash4.0 (Hamamatsu) camera. Recordings were controlled using MetaMorph 183 acquisition software. Data were processed in ImageJ and viewed as maximum-intensity 184 projections prior to analysis or figure preparation.

185

\section{Photo-ablation experiments}


187 Photo-ablations were performed with a Mai-Tai two-photon infrared laser (Spectra Physics)

188 attached to a Leica SP5 confocal microscope equipped with a 60X 1.3 NA objective with the 189 stage maintained at $25^{\circ} \mathrm{C}$. Z-series consisting of $10,1 \mu \mathrm{m}$ steps were acquired before and after 190 the photo-ablation at $30 \mathrm{sec}$ intervals. Photo-ablation was performed on the basal centrosome 191 using flies expressing GFP-H2A, PH-PLC $\delta$-GFP and Aurora A-GFP. Anaphase onset was 192 identified as the first signs of sister chromatid separation. The photo-ablation was considered 193 complete after the Aurora A signal on the basal centrosome could no longer be detected.

\section{Live cell imaging analysis}

196 Measurements of fluorescence intensities, mitotic spindle lengths and diameters of NB and 197 GMC cells were performed with ImageJ software ${ }^{35}$. The Sqh-GFP analyses were done on the 198 maximum projection of two optical sections $(1 \mu \mathrm{m})$. The polarity-dependent apical clearing 199 was calculated, as the first time point after anaphase onset, when myosin began to disappear

200 from the apical cortex ${ }^{36}$. NB cortex curvature analyses were performed according to 201 previously defined methods ${ }^{13}$. The furrow shift was determined as the distance between the 202 first ingression site and final cleavage site. To quantify the Myosin-GFP furrows width; a 203 segmented line was drawn along the NB half-cell cortex during anaphase and the GFP 204 intensity profiles were quantified along this line using ImageJ. The furrow width was 205 measured as the relative half-cell cortex length containing 60\% of the maximum Sqh-GFP signal intensity.

207

\section{Immunofluorescence analysis}

209 Larval brains from each genotype were processed for immunofluorescence studies as 210 described previously ${ }^{14}$. Briefly, wandering third instar larval brains from were dissected in 211 testis buffer (TB: $183 \mathrm{mM} \mathrm{KCL}, 47 \mathrm{mM} \mathrm{NaCl}, 10 \mathrm{MM}$ Tris, and $1 \mathrm{mM}$ EDTA, pH 6.8) and 212 brains were fixed for 20 minutes at $25^{\circ} \mathrm{C}$ in TBF (TB supplemented with $10 \%$ formaldehyde, 213 and $0.01 \%$ Triton X-100). Brains were then washed twice in PBS for 15 minutes, and twice in 214 PBS Triton X-100 0.1\% for 15 minutes. The brains were first incubated for 60 minutes at $21525^{\circ} \mathrm{C}$ in PBSTB (1\% BSA), before incubation with secondary antibodies. The samples were 216 observed with a SP5 confocal microscope (Leica) equipped with a 63X 1.4 NA objective lens. 217 Images are maximum intensity projections consisting of 4 optical sections acquired at $0.5 \mu \mathrm{m}$ 218 intervals. 


\section{Quantification of peripheral MTs in fixed NBs during mid anaphase}

221 Z-series were acquired every $0.2 \mu \mathrm{m}$ using a LSM 880 confocal microscope with Airyscan

222 (Zeiss) for telophase NBs. Images were then processed with the Zen software. Images were

223 analyzed with ImageJ as maximum intensity projections $(0.8 \mu \mathrm{m})$ consisting of 5 optical $(0.2$

$224 \mu \mathrm{m})$ sections in the plane of the furrow.

225

\section{Statistical analysis}

227 Differences between datasets were assessed with Prism 7.0a software (GraphPad), either by 228 non-parametric tests (Mann-Whitney-Wilcoxon) or parametric tests (Unpaired T). Non229 significance (ns) threshold was when $P>0.05$.

230

231

232

233

234

235

236

237 


\section{Results}

240 Cell size asymmetry is compromised following Ensconsin depletion but not overexpression in NBs

242 Neuroblasts divide asymmetrically to generate a large self-renewing neuroblast (NB) and a 243 smaller differentiating ganglion mother cell (GMC, Figure 1 A. We previously showed that

244 Ensconsin is required for MT polymerization during cell division; consequently ensc mutant 245 spindles are shorter than their wild type (WT) counterparts ${ }^{14}$. To investigate the possible 246 consequences of a change in spindle length on NB asymmetric cell division, we first 247 analyzed, by live cell imaging, cell size asymmetry of dividing NBs in wild type and ensc 248 mutants (Figure 1). We confirmed the previous finding that loss of Ensconsin triggered a $249 \sim 10 \%$ decrease in mitotic spindle length (Figure 1 B, C). Strikingly, the ensc mutants 250 displayed a small yet statistically significant reduction in the ratio between NB and GMC 251 diameters indicating a loss of asymmetry (Figure $1 \mathrm{D})$. This defect could either result from the 252 associated change in spindle length or indicate some uncharacterized function for Ensconsin 253 in asymmetrical size fate determination. To further explore the role of Ensconsin in MT 254 dynamics in vitro, we used TIRF microscopy and recombinant Ensconsin protein (Figure 1 255 E). Ensconsin-MBP had a small but significant effect on MT growth rate. Most striking was 256 the $\sim 50 \%$ reduction in the rate of MT shrinkage and the more than 3 times increase in the 257 rescue frequency compared to controls or MBP alone (Figure $1 \mathrm{~F}$ ). In line with these results, 258 over-expression of Ensconsin (Ensc-OE) in NBs lead to elongated spindles that buckled when 259 reaching the cortex (Figure $1 \mathrm{G}, \mathrm{H}, \mathrm{S} 1$ ), consistent with previous work in symmetrically 260 dividing S2 cells ${ }^{14}$. Despite the increase in MT polymerization and spindle length, the level 261 of size asymmetry remained unperturbed following cytokinesis in Ensc-OE NBs (Figure 1 I).

263 Enhancement of spindle length through over-expression of Msps or depletion of 264 Kinesin-8 MT depolymerase does not alter cell size asymmetry

265 To determine if daughter cell size asymmetry is insensitive to stimulation of MT growth, we 266 quantified size asymmetry following over-expression of the microtubule associated protein 267 Mini spindles, the fly orthologue of MAP215/ch-TOG, a protein with MT polymerization 268 properties ${ }^{37-39}$. In parallel, we performed RNAi-mediated depletion of the MT 269 depolymerizing Kinesin-8 fly family member Klp67A. This kinesin depolymerizes 270 microtubules and its depletion leads to the formation of exceptionally long spindles in 271 Drosophila cells ${ }^{40,41}$. Similar to Ensc-OE, over-expression of Msps-RFP (Msps-OE) or 
272 RNAi-mediated depletion of Klp67A led to the formation of long and bent mitotic spindles

273 (Figure 2 A, B and D; Figure S1; Video 1 and 2). Neither perturbation affected the post-

274 cleavage asymmetrical cell size (Figure 2A, C and E). These data suggest that asymmetric cell

275 size regulation is not sensitive to an increase in MT polymer or spindle length elongation.

276

277 Spindle shortening through over-expression of Kinesin-8 or $\mathbf{- 1 3}$ family MT 278 depolymerases decreases cell size asymmetry

279 To investigate if the size asymmetry reduction observed in ensc mutants (Figure 1) was 280 unique to Ensconsin or rather a common effect of spindle shortening, we induced other 281 perturbations of MT-polymerization by over-expressing two MT depolymerizing kinesins; 282 either Klp10A which belongs to the Kinesin-13 family (Klp10A-OE) or the Kinesin-8 283 member Klp67A (Klp67A-OE) (Figure S1). Importantly, whereas the depletion of either 284 causes spindle elongation, their over-expression results in abnormal shortening ${ }^{40,42-45}$. As 285 predicted both Klp10A-OE and Klp67A-OE NBs exhibited shorter spindles although the 286 length reduction was more pronounced in Klp10A-OE cells (Figure 2 F, G, I Video 3).

287 Depending on the spindle's length, it assumed a lesser or greater displacement relative to the 288 cell center. Interestingly, in these shortened spindle cells, like with ensc mutants, we found 289 that the NB/GMC diameter ratio was significantly impaired indicating that cell division was 290 more symmetric compared to controls (Figure $2 \mathrm{H}$ and J). Thus, defective MT polymerization 291 leading to spindle shortening due to loss of Ensconsin function or over-expression of the 292 Kinesin- 8s and 13s biases asymmetric cell division.

294 Defective MT growth leads to an apical shift of the basal cleavage furrow after anaphase 295 onset.

296 In asymmetrically dividing NBs, it was described that both polarity and midzone-dependent 297 mechanisms ensure that furrow components, including myosin, are positioned basally to 298 generate daughter cells of different sizes ${ }^{11,13,36}$. To investigate the behavior of the furrow, we 299 monitored the dynamics of the regulatory light chain of myosin in live NBs using Sqh-GFP ${ }^{46}$.

300 In control cells, we confirmed that Myosin-GFP was uniformly present at the cell cortex 301 before anaphase (Figure 3 A and Video 4). In ensc, Klp67A-OE and Klp10A-OE NBs, the 302 polarity-dependent step of myosin redistribution from the apical cortex was similar to 303 controls, in agreement with the perturbations not altering cell polarization (Figure S2 A and 304 B). We also analyzed the furrow positioning through curvature measurements of the cell 305 membrane $\left({ }^{13}\right.$ and Figure 3 B). While the furrow position remained stably placed from 
306

307

308

309

310

311

312

313

314

315

316

317

318

319

320

321

322

323

324

325

326

327

328

329

330

331

332

333

334

335

336

337

338

339

anaphase until cytokinesis in control NBs, we found it shifted significantly towards the apical side during completion of cell division in ensc and Klp67A-OE cells, with a maximal displacement observed for Klp10A-OE NBs (Figure 3, C Video 5 and 6). Moreover, while the furrow width was consistently $\sim 10 \%$ of the half-cell cortex length in control NBs as revealed by Myosin-GFP (Figure $3 \mathrm{D}$ and E), the signal occupied a larger space in ensc and Klp67AOE NBs with the maximum width of $\sim 25 \%$ of the half-cell cortex length observed for Klp10A-OE cells at comparable time points (Figure 3 A, E, Video 6). From this we conclude that proper MT growth is required for maintaining furrow size and position during asymmetrical cell division.

\section{Centralspindlin is spatially and temporally regulated as two distinct populations}

Our previous perturbations, which interfered with MT dynamics, suggested that a common mechanism was at play for maintaining the furrow position. In all higher eukaryotes examined to date, myosin recruitment and activation at the cleavage furrow is regulated by the highly conserved centralspindlin complex, a tetramer comprised of a Kinesin-6 family member complexed with Mgc-RacGAP (Pavarotti-klp and Tumbleweed in Drosophila, respectively) ${ }^{7}$ ${ }^{8,47}$. Strikingly, we found that the combination of Klp10A-OE and a single copy of the pav ${ }^{B 200}$ null allele enhanced the asymmetry defect observed with Klp10A-OE alone (Figure S2 C). Centralspindlin functionality and targeting to the membranes is regulated by the chromosomal passenger complex (CPC)-dependent oligomerization ${ }^{48}$. We therefore challenged the complex by introducing a single null allele for its Survivin subunit, $s v n^{2180}$, and monitored the effects on cell symmetry in the Klp10A-OE background. We found that Klp10A-OEdependent size asymmetry defects were further enhanced when Survivin levels were reduced (Figure S2 C). These results suggest that the observed asymmetry defects are due, at least in part, to impaired centralspindlin function.

In most eukaryotic cells, the centralspindlin complex is located at the spindle midzone and at the equatorial cortex. To characterize the furrow mis-positioning that accompanies defective microtubule growth, we analyzed the spatio-temporal distribution of the motor component of centralspindlin, Pavarotti-klp, in different experimental backgrounds. We began by examining GFP-Pav-klp ${ }^{21}$ localization in control NBs. Our time-lapse studies showed that most of the GFP-Pav-klp was located at the cortex at the cleavage site. Following the onset of furrow ingression, a second pool started to accumulate into a small and spatially distinct band near the former site occupied by the metaphase chromosomes at the spindle midzone (Figure $4 \mathrm{~A}$, Figure S3A, and Video 7). The spatial and temporal separation of the GFP-Pav-klp signals led 
340 us to speculate that these were separate pools of centralspindlin. To confirm this hypothesis,

341 we tracked GFP-Pav-klp in cells lacking MTs that were forced into anaphase using Mad2

342 RNAi to abrogate the spindle assembly checkpoint ${ }^{14}$. Under these conditions GFP-Pav-klp

343 showed a slight enrichment at the basal cortex but this pool remained at almost baseline levels

344 compared to control cells, which showed continuous recruitment of GFP-Pav-klp following

345 anaphase onset (Figure S3 B and C). When microtubule polymerization was impaired in ensc,

346 Klp10A-OE and Klp67A-OE cells, even if the centralspindlin component GFP-Pav-klp was

347 initially present at the equatorial cell cortex, it did not become enriched at the cleavage site to

348 the levels measured in controls (Figure 4 B, C, E red triangles, Figure S4 A, see also Video 8

349 and 9). Instead, in Klp10A-OE (Video 9), Klp67A-OE but not in ensc NBs (Video 8), GFP-

350 Pav-klp appeared more abundant at the spindle midzone (Figure $4 \mathrm{~B}$ and $\mathrm{C}$, see time $100 \mathrm{~s}$

351 blue arrows and insets at time $180 \mathrm{sec}$, Figure $4 \mathrm{E}$ and Figure S4 B). Together, these

352 experiments show that centralspindlin exists as two distinct and separable populations, one at

353 the basal cortex and one at the spindle midzone. The decrease of the cortical centralspindlin

354 pool is always accompanied by a displacement of the cleavage furrow.

355

\section{The spatio-temporal regulation of centralspindlin relies on stable peripheral MTs}

357 Fluorescence quantification (Figure $4 \mathrm{~F}$ ) revealed that compared to wild type, Klp10-OE and

358 Klp67A-OE NBs both displayed a decrease in cortical GFP-Pav-klp signal with a concomitant

359 increase at the midzone (Figure S4). To further characterize the relationship between cortical

360 and midzone centralspindlin pools and the role of MT growth in asymmetrical cleavage, we

361 examined GFP-Pav-klp dynamics in sas $4^{\text {s2214 }}$ mutants, which lack centrosomes and their

362 associated astral MTs ${ }^{28}$. In this background, cortical enrichment also appeared diminished

363 relative to the midzone (Figure 4 D, Figure S4 A and B). An enlarged view of the boxed

364 regions for $180 \mathrm{sec}$ post-anaphase onset highlights this increased centralspindlin recruitment

365 at the spindle midzone and weaker accumulation at the cell cortex (Figure 4 E). Although

366 sas $4^{\text {s2214 }}$ NBs exhibited signal enrichment at their midzones, not all cells had a clear cortical

367 reduction (Figure S4). Strikingly, $s a s 4^{\text {s2214 }}$ mutants exhibited both an increased spindle length

368 as well as a significant overall cell size asymmetry defect (Figure S5 A and B), further

369 supporting the idea that astral MTs maintain basal furrow position. To confirm the

370 contribution of the MT-aster in furrow positioning and maintenance, we removed it by laser

371 ablation of the basal centrosome. Each centrosome was labeled with GFP-tagged Aurora A

372 and basal proximal centrosome was ablated by a multi-photon laser until the signal was no

373 longer detectable. Consistent with centrosome removal, ablated cells displayed a phenotype 
374 virtually identical to sas $4^{\text {s2214 }}$ mutants: daughter cells exhibiting cell size asymmetry after the

375 ensuing cytokinesis (Figure S5 C and D, Video 10, compare left and right). These live cell

376 observations suggested that astral MTs were essential to furrow positioning. We therefore

377 performed a quantitative analysis of fixed preparations examining MT distribution during

378 early telophase. Detailed morphological examination revealed that in wild type NBs bundles

379 of astral MTs capped the future ganglion mother cell and spread apically, closely apposed to

380 the cortex at the cleavage furrow. This was not the case with ensc, Klp67A-OE or Klp10-OE

381 NBs, which showed decreased MT densities and lacked the presumptive bundles (Figure S5 E

382 and F). Altogether, our data strongly suggest that peripheral astral MTs originating from the

383 basal centrosome, in a close vicinity of the basal furrow play a key role in accurate

384 asymmetric cell division.

385

386 The early spindle midzone and furrow occupy distinct positions in NBs

387 The presence of GFP-Pav-klp at the spindle midzone distal to the cleavage site and the

388 movement of the furrow towards the equator in peripheral MTs-deficient cells prompted us to

389 further characterize the cleavage site and the midzone in wild type cells. For this purpose, we

390 used Fascetto-GFP (the homologue of the mammalian PRC1 protein; Feo-GFP) a marker that

391 uniquely labels the spindle midzone (Figure 5 A) ${ }^{27,49}$. We found that in these NBs the

392 metaphase plate was slightly shifted toward the basal side relative to the cell equator along the

393 apico-basal axis (Figure $5 \mathrm{~A},-120 \mathrm{sec}$, arrowhead and Figure $5 \mathrm{C}$ ) but similarly placed to the

394 midzone-defining Feo-GFP signal that appears following anaphase chromosome segregation

395 (Figure $5 \mathrm{~A}$, time $90 \mathrm{sec}$, and Figure $5 \mathrm{D}$ arrowhead). This was in contrast to the position of

396 the furrow (Figure 5 A, time 90, compare green and white arrows; E arrowhead). Indeed,

397 while the metaphase plate and midzone were interchangeably located, the furrow was always

398 distinct and basally distal to these (Figure 5 F, Video 11). Interestingly, kymograph analyses

399 of the spindle midzone and cell membranes reveals that the midzone moves basally during the

400 ingression of the furrow until they ultimately consolidate into a single structure (Figure 5 B).

401 In summary, these data demonstrate that the spindle midzone in wild type cells occupies a

402 spatially different position than that of the furrow and its associated cortical MTs. Both of

403 these can recruit centralspindlin, however, under normal circumstances it is the cortical pool

404 that dominates in Drosophila NBs to define the cleavage site. 
405

406

407

408

409

410

411

412

413

414

415

416

417

418

419

420

421

422

423

424

425

426

427

428

429

430

431

432

433

434

435

436

437

438

\section{Discussion}

Asymmetric cell division is a robust process that ensures that two daughter cells inherit different fates and sizes. The Drosophila NB is a powerful and widely used model system to study this specialized form of division because of the large number of NBs in the developing Drosophila brain, rapid division time and experimental traceability ${ }^{50-52}$. Moreover, although these cells are relatively small, they are highly asymmetrical following cytokinesis allowing accurate measurements and analyses. In this study, we have used this model system to challenge asymmetric cell division after modification of MT growth dynamics. We were able to increase mitotic spindle length using over-expression of MT polymerizing MAPs (Msps and Ensconsin), as well as by RNAi-mediated depletion of Klp67A, a member belonging to Kinesin-8 family of MT depolymerizing Kinesins. Despite the presence of long and bent mitotic spindles under these conditions, the NB cell size ratio remained unchanged relative to wild type NBs. This reveals that asymmetric cell division and asymmetric positioning of the cleavage furrow are resistant to an excess of abnormally long and stable MTs during cell division. By contrast, decreasing MT stability and shortening of the mitotic spindle produced more symmetric cell divisions. This change was due to an apical shift of the cleavage furrow during its ingression. This phenotype was not MAP specific since over-expression of either Klp10A (Kinesin-13) or Klp67A (Kinesin-8) MT depolymerases, as well as the deletion of Ensconsin produced similar effects. Rather they suggest that spindle size or interference with microtubule dynamics is responsible for the phenotype. Interestingly, sas-4 $4^{s 2214}$ mutants which are reported to lack functional centrosomes and thus astral microtubules yielded reduced levels of cell size asymmetry although these NBs harbored longer mitotic spindles ${ }^{28}$. This reveals that astral MTs and not the mitotic spindle length is the key element responsible for the level of size asymmetry observed in NBs. Consistent with this hypothesis, loss of the basal MT-aster, through targeted laser irradiation and ablation prior anaphase onset, also reduced sibling cell size asymmetry. Taken together these results strongly suggest that a population of basal peripheral astral MTs is required to maintain a cleavage site, which normally favors a basal position in the fly neuroblast. In agreement with this our quantification of peripheral MT bundles close to the ingression furrow revealed that they are significantly decreased during telophase in ensc, Klp10A-OE and Klp67A-OE NBs. Our results are in accord with reports indicating that a subpopulation of stable astral MTs play a key role in the initiation of furrowing in symmetrically dividing cells and that in some systems, furrowing can occur without the presence of a stable central spindle ${ }^{3,6,7,20,53-56}$. 
439 However in contrast to other studies which utilize micromanipulation and laser ablation, our 440 data reveals that in wild type asymmetrically dividing NBs, the astral MT furrowing pathway 441 dominates over the midzone pathway. Previous studies have suggested that NBs have two 442 genetically separable pathways to drive cytokinesis. The first, cortical polarity pathway, is 443 responsible for the targeting of the furrowing machinery to the basal cortex on the surface of 444 what is destined to become the smaller differentiating ganglion mother cell ${ }^{11,12}$. Interference 445 with this polarity pathway prevents myosin enrichment at the basal cortex, leading to 446 symmetric division. The alternative spindle pathway relies on the spindle midzone and the 447 chromosomal passenger complex ${ }^{9},{ }^{12}$. However, several of our observations are at odds with 448 these previous findings and favor a model of furrow positioning that relies largely on 449 peripheral astral MTs with little if any contribution from the spindle midzone; (i) live cell 450 imaging and analyses utilizing GFP-Pav-klp as a marker of centralspindlin position revealed 451 that this master controller of cytokinesis accumulated at the basal cortex throughout the entire 452 furrow ingression process. (ii) Centralspindlin levels were low at the midzone during furrow 453 placement and ingression compared to the cortex (Figure 4). (iii) We consistently observed 454 that the midzone, as defined independently using both GFP-Pav-klp and Feo-GFP, was 455 spatially independent from the furrowing site (Figures 5B, 6A). In addition, the midzone 456 ultimately moved to the position of the furrow and not vice-versa (Figure 5B), confirming 457 previous observations in embryonic NBs ${ }^{57}$. (iv) Finally, genetic or photo-based removal of 458 the basal centrosome precluded peripheral MT formation and interaction with the cortex. 459 Accordingly, the cortical centralspindlin pool was diminished and cells experienced a size 460 asymmetry defect (Figure 4and S4).

461 Our localization studies employing Feo-GFP suggest that under normal conditions midzone462 associated centralspindlin does not perform a key role in positioning of the cleavage site and 463 that this function is served by the more abundant centralspindlin pool associated with the 464 membranes at the cleavage site. However, when peripheral MTs were impaired, 465 centralspindlin enrichment at the furrow was diminished, leading to a decreased 466 midzone/furrow centralspindlin ratio and a reset of the furrowing toward the equatorial 467 midzone. This indicates that the two populations of centralspindlin are competent to signal 468 furrowing but that the cortical pool delivered by astral MTs is normally dominant. Thus the 469 spatial localization and the cortical/midzone ratio of centralspindlin are the pivotal 470 determinants of final furrow positioning in the Drosophila NB. Interestingly, a recent study 471 has shown that a similar competition between centralspindlin pools also occurs in human cells $472 \quad 58$, revealing an evolutionary conservation of the mechanism. 
473 As with human cells, we found that the CPC activity seems essential in this regulatory event

474 (Figure S2 C). In contrast to a recent study in S2 cells, we do not observe GFP-Pav-klp

475 labelling at the plus ends of astral MTs ${ }^{59}$ even when studied by enhanced resolution imaging

476 methods. Instead, we consistently find that centralspindlin coats the entire length of astral

477 MTs, suggesting that the plus end directed motor activity of Pav-klp is used to bring

478 centralspindlin to the furrow in Drosophila NBs in agreement with previous studies in early

479 embryos ${ }^{21,60}$.

480 Altogether, our data suggest a model in which a competition between different centralspindlin

481 populations is a key determinant of asymmetric division in Drosophila NBs. The concerted

482 and consecutive action of polarity cues and astral MTs as centralspindlin delivery arrays are

483 essential in this process. The ability of the spindle midzone to define furrow and cleavage

484 location only becomes engaged during late telophase and after subcortical astral MTs are

485 compromised. Despite their clear role in governing size asymmetry, we have not been able to

486 induce complete daughter cell size equality through any of a host of MT perturbing

487 treatments. This suggests that additional feedback mechanisms exist to ensure a threshold

488 level of asymmetry in these cells. Elucidating these systems and their evolutionary advantages

489 will be important directions for future investigations. 


\section{Acknowledgments}

492

493 We thank Gregory Rogers, Pier Paolo d'Avino, Renata Basto, Gohta Goshima, Jordan Raff,

494 Hiro Ohkura, Anne Royou, Roger Karess, Christian Dahmann, Antoine Guichet, Juliette

495 Mathieu, Jean-René Huynh, Clemens Cabernard, Tri Pham for providing fly stocks,

496 antibodies and cDNAs. We thank Chloé Rauzier for preliminary functional analyzes of Ensc-

497 OE and Msps-OE NBs. This work was funded by the Ligue Nationale Contre le Cancer, the

498 Fondation ARC pour la Recherche sur le Cancer. A. T. is a doctoral fellow of the Région

499 Bretagne and the Ligue Nationale contre le Cancer. We thank the Photonic Imaging Center of

500 Grenoble Institute of Neurosciences, which is part of ISdV core facility. We thank Xavier

501 Pinson, Stéphanie Dutertre and Sébastien Huet for advices and help with the microscopes and

502 the Microscopy Rennes Imaging Center platform. We thank Romain Gibeaux, Pier Paolo

503 d'Avino and Christelle Benaud for ideas, critical readings and helpful suggestions. The

504 authors have no competing financial interests to declare. 


\section{References}

1. von Dassow, G. Concurrent cues for cytokinetic furrow induction in animal cells. myosin activation zone during cytokinetic furrow formation. J Cell Biol 183, 457-470 (2008).

4. Vale, R.D., Spudich, J.A. \& Griffis, E.R. Dynamics of myosin, microtubules, and Kinesin-6 at the cortex during cytokinesis in Drosophila S2 cells. J Cell Biol 186, 727 738 (2009).

5. Shannon, K.B., Canman, J.C., Ben Moree, C., Tirnauer, J.S. \& Salmon, E.D. Taxolstabilized microtubules can position the cytokinetic furrow in mammalian cells. $\mathrm{Mol}$ Biol Cell 16, 4423-4436 (2005).

6. Bringmann, H. \& Hyman, A.A. A cytokinesis furrow is positioned by two consecutive signals. Nature 436, 731-734 (2005).

7. Mishima, M. Centralspindlin in Rappaport's cleavage signaling. Seminars in cell \& developmental biology 53, 45-56 (2016).

8. D'Avino, P.P., Giansanti, M.G. \& Petronczki, M. Cytokinesis in animal cells. Cold Spring Harbor perspectives in biology 7, a015834 (2015).

9. Glotzer, M. Cytokinesis in Metazoa and Fungi. Cold Spring Harbor perspectives in biology 9 (2017).

10. Knoblich, J.A. Asymmetric cell division: recent developments and their implications for tumour biology. Nat Rev Mol Cell Biol 11, 849-860 (2010).

11. Cabernard, C., Prehoda, K.E. \& Doe, C.Q. A spindle-independent cleavage furrow positioning pathway. Nature 467, 91-94 (2010).

12. Roth, M., Roubinet, C., Ifflander, N., Ferrand, A. \& Cabernard, C. Asymmetrically dividing Drosophila neuroblasts utilize two spatially and temporally independent cytokinesis pathways. Nat Commun 6, 6551 (2015).

13. Tsankova, A., Pham, T.T., Garcia, D.S., Otte, F. \& Cabernard, C. Cell Polarity Regulates Biased Myosin Activity and Dynamics during Asymmetric Cell Division via Drosophila Rho Kinase and Protein Kinase N. Dev Cell 42, 143-155 e145 (2017).

14. Gallaud, E. et al. Ensconsin/Map7 promotes microtubule growth and centrosome separation in Drosophila neural stem cells. J Cell Biol 204, 1111-1121 (2014).

15. Metivier, M. et al. Dual control of Kinesin-1 recruitment to microtubules by Ensconsin in Drosophila neuroblasts and oocytes. Development 146 (2019).

16. Widmann, T.J. \& Dahmann, C. Dpp signaling promotes the cuboidal-to-columnar shape transition of Drosophila wing disc epithelia by regulating Rho1. J Cell Sci 122, 1362-1373 (2009).

17. Bischof, J. et al. A versatile platform for creating a comprehensive UAS-ORFeome library in Drosophila. Development 140, 2434-2442 (2013).

18. Dietzl, G. et al. A genome-wide transgenic RNAi library for conditional gene inactivation in Drosophila. Nature 448, 151-156 (2007).

19. Royou, A., Field, C., Sisson, J.C., Sullivan, W. \& Karess, R. Reassessing the role and dynamics of nonmuscle myosin II during furrow formation in early Drosophila embryos. Mol Biol Cell 15, 838-850 (2004).

20. Inoue, Y.H. et al. Mutations in orbit/mast reveal that the central spindle is comprised of two microtubule populations, those that initiate cleavage and those that propagate furrow ingression. J Cell Biol 166, 49-60 (2004). 
554 21. Minestrini, G., Harley, A.S. \& Glover, D.M. Localization of Pavarotti-KLP in living Drosophila embryos suggests roles in reorganizing the cortical cytoskeleton during the mitotic cycle. Mol Biol Cell 14, 4028-4038 (2003).

558

22. Adams, R.R., Tavares, A.A., Salzberg, A., Bellen, H.J. \& Glover, D.M. pavarotti encodes a kinesin-like protein required to organize the central spindle and contractile ring for cytokinesis. Genes Dev 12, 1483-1494 (1998).

23. Mathieu, J. et al. Aurora B and cyclin B have opposite effects on the timing of cytokinesis abscission in Drosophila germ cells and in vertebrate somatic cells. Dev Cell 26, 250-265 (2013).

24. Claret, S., Jouette, J., Benoit, B., Legent, K. \& Guichet, A. PI(4,5)P2 produced by the PI4P5K SKTL controls apical size by tethering PAR-3 in Drosophila epithelial cells. Curr Biol 24, 1071-1079 (2014).

25. Gervais, L., Claret, S., Januschke, J., Roth, S. \& Guichet, A. PIP5K-dependent production of PIP2 sustains microtubule organization to establish polarized transport in the Drosophila oocyte. Development 135, 3829-3838 (2008).

26. Caous, R. et al. Spindle assembly checkpoint inactivation fails to suppress neuroblast tumour formation in aurA mutant Drosophila. Nat Commun 6, 8879 (2015).

27. Wang, H., Brust-Mascher, I. \& Scholey, J.M. The microtubule cross-linker Feo controls the midzone stability, motor composition, and elongation of the anaphase B spindle in Drosophila embryos. Mol Biol Cell 26, 1452-1462 (2015).

28. Basto, R. et al. Flies without centrioles. Cell 125, 1375-1386 (2006).

29. Hyman, A. et al. Preparation of modified tubulins. Methods Enzymol 196, 478-485 (1991).

30. Ramirez-Rios, S. et al. A TIRF microscopy assay to decode how tau regulates EB's tracking at microtubule ends. Methods Cell Biol 141, 179-197 (2017).

31. Lee, M.J., Gergely, F., Jeffers, K., Peak-Chew, S.Y. \& Raff, J.W. Msps/XMAP215 interacts with the centrosomal protein D-TACC to regulate microtubule behaviour. Nat Cell Biol 3, 643-649 (2001).

32. Goshima, G. \& Vale, R.D. Cell cycle-dependent dynamics and regulation of mitotic kinesins in Drosophila S2 cells. Mol Biol Cell 16, 3896-3907 (2005).

33. Rogers, S.L., Rogers, G.C., Sharp, D.J. \& Vale, R.D. Drosophila EB1 is important for proper assembly, dynamics, and positioning of the mitotic spindle. J Cell Biol 158, 873-884 (2002).

34. Jordan, P. \& Karess, R. Myosin light chain-activating phosphorylation sites are required for oogenesis in Drosophila. J Cell Biol 139, 1805-1819 (1997).

35. Rueden, C.T. et al. ImageJ2: ImageJ for the next generation of scientific image data. BMC bioinformatics 18, 529 (2017).

36. Roubinet, C. et al. Spatio-temporally separated cortical flows and spindle geometry establish physical asymmetry in fly neural stem cells. Nat Commun 8, 1383 (2017).

37. Reber, S.B. et al. XMAP215 activity sets spindle length by controlling the total mass of spindle microtubules. Nat Cell Biol 15, 1116-1122 (2013).

38. Cullen, C.F., Deak, P., Glover, D.M. \& Ohkura, H. mini spindles: A gene encoding a conserved microtubule-associated protein required for the integrity of the mitotic spindle in Drosophila. J Cell Biol 146, 1005-1018 (1999).

39. Fox, J.C., Howard, A.E., Currie, J.D., Rogers, S.L. \& Slep, K.C. The XMAP215 family drives microtubule polymerization using a structurally diverse TOG array. Mol Biol Cell 25, 2375-2392 (2014).

40. Goshima, G., Wollman, R., Stuurman, N., Scholey, J.M. \& Vale, R.D. Length control of the metaphase spindle. Curr Biol 15, 1979-1988 (2005). 
603 41. Edzuka, T. \& Goshima, G. Drosophila kinesin-8 stabilizes the kinetochore-

604

605

606

607

608

609

610

611

612

613

614

615

616

617

618

619

620

621

622

623

624

625

626

627

628

629

630

631

632

633

634

635

636

637

638

639

640

641

642

643

644

645

646

647

648

649

650

651

652 microtubule interaction. J Cell Biol 218, 474-488 (2019).

42. Laycock, J.E., Savoian, M.S. \& Glover, D.M. Antagonistic activities of Klp10A and Orbit regulate spindle length, bipolarity and function in vivo. J Cell Sci 119, 23542361 (2006).

43. Morales-Mulia, S. \& Scholey, J.M. Spindle Pole Organization in Drosophila S2 Cells by Dynein, Abnormal Spindle Protein (Asp), and KLP10A. Mol Biol Cell 16, 31763186 (2005).

44. Radford, S.J., Harrison, A.M. \& McKim, K.S. Microtubule-depolymerizing kinesin KLP10A restricts the length of the acentrosomal meiotic spindle in Drosophila females. Genetics 192, 431-440 (2012).

45. Buster, D.W., Zhang, D. \& Sharp, D.J. Poleward tubulin flux in spindles: regulation and function in mitotic cells. Mol Biol Cell 18, 3094-3104 (2007).

46. Royou, A., Sullivan, W. \& Karess, R. Cortical recruitment of nonmuscle myosin II in early syncytial Drosophila embryos: its role in nuclear axial expansion and its regulation by Cdc2 activity. J Cell Biol 158, 127-137 (2002).

47. D'Avino, P.P., Savoian, M.S. \& Glover, D.M. Cleavage furrow formation and ingression during animal cytokinesis: a microtubule legacy. J Cell Sci 118, 1549-1558 (2005).

48. Basant, A. et al. Aurora B kinase promotes cytokinesis by inducing centralspindlin oligomers that associate with the plasma membrane. Dev Cell 33, 204-215 (2015).

49. Verni, F. et al. Feo, the Drosophila homolog of PRC1, is required for central-spindle formation and cytokinesis. Curr Biol 14, 1569-1575 (2004).

50. Rusan, N.M. \& Peifer, M. A role for a novel centrosome cycle in asymmetric cell division. J Cell Biol 177, 13-20 (2007).

51. Januschke, J. \& Gonzalez, C. Drosophila asymmetric division, polarity and cancer. Oncogene 27, 6994-7002 (2008).

52. Rebollo, E. et al. Functionally unequal centrosomes drive spindle orientation in asymmetrically dividing Drosophila neural stem cells. Dev Cell 12, 467-474 (2007).

53. Murthy, K. \& Wadsworth, P. Dual role for microtubules in regulating cortical contractility during cytokinesis. J Cell Sci 121, 2350-2359 (2008).

54. Strickland, L.I., Donnelly, E.J. \& Burgess, D.R. Induction of cytokinesis is independent of precisely regulated microtubule dynamics. Mol Biol Cell 16, 44854494 (2005).

55. Canman, J.C. et al. Determining the position of the cell division plane. Nature 424, 1074-1078 (2003).

56. Kotynkova, K., Su, K.C., West, S.C. \& Petronczki, M. Plasma Membrane Association but Not Midzone Recruitment of RhoGEF ECT2 Is Essential for Cytokinesis. Cell reports 17, 2672-2686 (2016).

57. Kaltschmidt, J.A., Davidson, C.M., Brown, N.H. \& Brand, A.H. Rotation and asymmetry of the mitotic spindle direct asymmetric cell division in the developing central nervous system. Nat Cell Biol 2, 7-12 (2000).

58. Adriaans, I.E., Basant, A., Ponsioen, B., Glotzer, M. \& Lens, S.M.A. PLK1 plays dual roles in centralspindlin regulation during cytokinesis. J Cell Biol 218, 1250-1264 (2019).

59. Verma, V. \& Maresca, T.J. Microtubule plus-ends act as physical signaling hubs to activate RhoA during cytokinesis. eLife 8 (2019).

60. Minestrini, G., Mathe, E. \& Glover, D.M. Domains of the Pavarotti kinesin-like protein that direct its subcellular distribution: effects of mislocalisation on the tubulin and actin cytoskeleton during Drosophila oogenesis. J Cell Sci 115, 725-736 (2002). 


\section{Legend to the figures}

656

657

Figure 1. Analysis of cell size asymmetry in ensc and Ensc-OE NBs

658 A) Scheme of a NB during cell division during metaphase (left) and telophase (right). Note 659 that the NB cell division is asymmetric and produces a large NB and a small Ganglion Mother 660 Cell (GMC) during cytokinesis. B) Selected images of a control NB (top) and an ensc mutant 661 NB (bottom) during cell division. The membranes are displayed in green and the MTs are 662 displayed in magenta. Time is min:s. Scale bar: $10 \mu \mathrm{m}$. C) Dot plot showing the mitotic 663 spindle length/NB diameter ratio ( \pm s.d.) in control $(0.86 \pm 0.05, n=27)$ or in ensc NBs $664(0.79 \pm 0.06, n=23), * * *: \quad P<0.0001$ (Wilcoxon test). D) Dot plot showing the NB diameter/GMC diameter ratio ( \pm s.d.) in control $(2.29 \pm 0.20, n=27)$ or in ensc NBs $(2.16 \pm 0.20$, $n=23$ ), *: $P<0.05$ (Wilcoxon test). E) Kymographs showing microtubules assembled from

667 GMPCPP seeds and $14 \mu \mathrm{M}$ tubulin in absence or in presence of $200 \mathrm{nM}$ of MBP or MBP-

668 Ensconsin. Horizontal and vertical scale bar are $5 \mu \mathrm{m}$ and 60 s respectively. F) Graphs 669 showing the growth and shrinkage rates and the catastrophe and rescue frequencies 670 determined from kymographs shown in E. ns: non significant; $* * * *: P<0.0001$ (Kruskal-

671 Wallis ANOVA followed by post-hoc Dunn's multiple comparison, total number of growth events $=116,123$ and 107, shrinkage events $=67,73$ and 81, catastrophe events $=94,96,83$ and rescue events $=3,3$ and 33 for the control, MPB and MPB-ensconsin respectively). G) 674 Selected images of a control NB (top) and an Ensc-OE NB (bottom) during cell division. 675 Membranes are displayed in magenta and MTs or Ensconsin are displayed in green. H) Dot 676 plot showing the mitotic spindle length/NB diameter ratio ( \pm s.d.) in control $(0.86 \pm 0.06$, $677 n=25)$ or in Ensc-OE NBs $(0.95 \pm 0.08, n=19), * * * *: P<0.0001$ (Wilcoxon test). I) Dot plot 678 showing the NB diameter/GMC diameter ratio ( \pm s.d.) in control $(2.36 \pm 0.17, n=25)$ or in 679 Ensc-OE NBs $(2.40 \pm 0.19, n=19)$, ns: non-significant (Wilcoxon test) J) Summary of NB 680 division in ensc (left) or Ensc-OE (right). ensc mutant NBs display shorter spindles and 681 undergo less asymmetric cell division while Ensc-OE NBs, despite harboring long spindles, 682 divides asymmetrically similar to WT. Time is min:s. 
686 A) Selected images of control (top), Msps-OE (middle) and Klp67A RNAi (bottom) NBs. The 687 membranes are shown in green, the MTs (top and bottom) and Msps (middle) are shown in 688 magenta. Time is min:s. Scale bar: $10 \mu \mathrm{m}$. B) Dot plot showing the mitotic spindle length/NB 689 diameter ratio ( \pm s.d.) in the NB of control $(0.86 \pm 0.06, n=18)$ or in Msps-OE transgenic flies 690 (1.03 $\pm 0.1, n=23$ ), $* * * *: \quad P<0.0001$ (Wilcoxon test). C) Dot plot showing the NB 691 diameter/GMC diameter ratio ( \pm s.d.) in control $(2.34 \pm 0.16, n=18)$ or in Msps-OE NBs $692(2.29 \pm 0.28, n=21)$, ns: non-significant (Wilcoxon test). D) Dot plot showing the mitotic 693 spindle length/NB diameter ratio ( \pm s.d.) in control NBs $(0.83 \pm 0.07, n=40)$ or in $K l p 67 A$ 694 RNAi NBs $(1.19 \pm 0.15, n=30), * * * *: P<0.0001$ (Wilcoxon test). E) Dot plot showing the NB 695 diameter/GMC diameter ratio in control $(2.31 \pm 0.16, n=40)$ or in Klp67A RNAi NBs 696 (2.32 $\pm 0.23, n=30)$, ns: non-significant (Wilcoxon test). F) Selected images of control (top), 697 Klp10A-OE (middle) and Klp67A-OE (bottom) NBs. The membranes are shown in green, the 698 MTs are shown in magenta. Scale bar: $10 \mu \mathrm{m}$. Time is min:s. G) Dot plot showing the mitotic 699 spindle length/NB diameter ratio ( \pm s.d.) in control NBs $(0.79 \pm 0.08, n=49)$ or in Klp10A-OE 700 NBs $(0.63 \pm 0.09, n=49), * * * *: P<0.0001$ (Wilcoxon test). H) Dot plot showing the NB 701 diameter/GMC diameter ratio ( \pm s.d.) in control NBs $(2.29 \pm 0.17, n=49)$ or in Klp10A-OE 702 NBs $(2.00 \pm 0.27, n=49)$, ****: $P<0.0001$ (Wilcoxon test). I) Dot plot showing the mitotic 703 spindle length/NB diameter in control NBs $(0.83 \pm 0.07, n=40)$ or in Klp67A-OE NBs $704(0.73 \pm 0.08, n=48)$, ****: $P<0.0001$ (Wilcoxon test). J) Dot plot showing the NB 705 diameter/GMC diameter ratio in control NBs $(2.32 \pm 0.16, n=40)$ or in Klp67A RNAi NBs $706 \quad(2.19 \pm 0.18, n=48), * * * *: P<0.0001$ (Wilcoxon test).

707

708 Figure 3. Analysis of myosin dynamics and furrow positioning in ensc, Klp67-OE and 709 KIp10A-OE

710 A) Selected images of (from top to bottom) dividing control, ensc, Klp67A-OE and Klp10A711 OE NBs expressing tubulin (magenta) and myosin regulatory light chain (green and lower 712 panels in monochrome) after anaphase onset ( $\mathrm{t}=20 \mathrm{~s})$ till late telophase. Scale bar: $10 \mu \mathrm{m}$. Time 713 is s. B) Scheme showing the possible apical shift between the initial and final furrow 714 curvature analysis. C) Dot plot showing of the relative furrow displacement between early 715 anaphase and late telophase in control $(0.00 \pm 0.04, n=18)$, ensc $(-0.03 \pm 0.05, n=18)$, Klp67A716 OE $(-0.05 \pm 0.04, n=12)$, and Klp10A-OE NBs $(-0.14 \pm 0.04, n=22)$. *: $P<0.05$, ***: $P<0.001$, $717 * * * *: P<0.0001$ (Wilcoxon test). D) Scheme showing the furrow width (red) during mid 718 anaphase. E) Dot plot showing the relative myosin furrow width/cell length ratio for control 
$719(0.08 \pm 0.02, n=18)$, ensc $(0.14 \pm 0.07, n=17)$, Klp67A-OE $(0.13 \pm 0.05, \mathrm{n}=12)$, and Klp10A-OE

720 NBs $(0.25 \pm 0.11, n=23), *: P<0.05, * * *: P<0.001, * * * *: P<0.0001$ (Wilcoxon test)).

721

722 Figure 4. Analysis of centralspindlin localization and dynamics in control, ensc, Klp10A-

\section{OE and sas $-4^{\mathrm{s} 2214} \mathrm{NBs}$}

724 A) Selected images of dividing control expressing tubulin (magenta) and GFP-Pav-klp (green

725 and lower panels in monochrome) from anaphase onset till late telophase (top left). B) ensc

726 NB. C) Klp10A-OE NB. D) $s a s-4^{\text {s2214 }}$ NBs (bottom). Scale bar: $10 \mu \mathrm{m}$. Time is s. E) Higher 727 magnification view of the selected control ensc, Klp10A-OE and $s a s-4^{s 2214}$ telophase NBs 728 (from panels in A-D) showing GFP-Pav-klp localization at the cleavage site. See the strong 729 signal at the cell cortex (red arrowheads) and the weak signal at the presumptive spindle

730 midzone away from the cleavage site, toward the apical side blue (blue arrows). F) Scheme of 731 the cleavage site showing the cortical and midzone centralspindlin pools. G) Dot plot ( \pm s.d.)

732 showing the relative cortical/midzone GFP intensity ratio for control $(2.57 \pm 1.13, n=15)$, ensc

$733(1.75 \pm 0.52, n=11)$, Klp10A-OE $(1.20 \pm 0.53, n=12)$, sas $-4^{s 2214}(1.24 \pm 0.54, n=10)$, control $734(4.2 \pm 1.76, n=7)$ and Klp67A-OE NBs $(2.17 \pm 1.15, n=11) . *: P<0.05, * * *: P<0.001$, ****: $735 P<0.0001$ (Wilcoxon test).

737 Figure 5. Analysis of the spindle midzone and the cleavage furrow position in brain NBs during cell division

739 A) Selected images of a WT NB expressing Feo-GFP (green and lower panels in 740 monochrome), MTs (magenta), and membranes during cell division (green and lower panels 741 in monochrome). The metaphase plate is indicated by a white arrowhead (-120s). The spindle 742 midzone is indicated by red arrowheads and the furrow is indicated by green arrowheads (60743 300s). Scale bar: $10 \mu \mathrm{m}$. Time is s. B) Kymograph showing the localization of the spindle 744 midzone and the cell contours during the time course of the NB cell division shown in panel 745 A, along the apico-basal axis. C) Dot Plot ( \pm s.d.) showing the mean relative metaphase plate 746 position along the apico-basal cortex $90 \mathrm{sec}$ after anaphase onset $(0.56 \pm 0.02 n=21)$. D) Dot 747 Plot showing the mean relative spindle midzone position ( \pm s.d.) along the apico-basal cortex $74890 \mathrm{sec}$ after anaphase onset $(0.59 \pm 0.02, n=23)$. E) Dot Plot $( \pm$ s.d. $)$ showing the mean relative 749 furrow position along the apico-basal cortex $90 \mathrm{sec}$ after anaphase onset $(0.72 \pm 0.13, n=23)$. F)

750 Dot Plot ( \pm s.d.) showing the mean relative distance between the furrow position and the 751 spindle midzone $90 \mathrm{sec}$ after anaphase onset $(0.13 \pm 0.02, n=23)$. 
753 Figure S1. Western Blot analyses of Ensconsin, Minispindles, Klp10A, Klp67A protein 754 in brain extracts

755 Brain subjected to RNAi or overexpression (OE) for the indicated MAPs were dissected and

756

757

758

759

760

761

762

763

764

765

766

767

768

769

770

771

772

773

774

775

776

777

778

779

780

781

782

783

784

785

786

resuspended in sample buffer before for Western blot analyses. The primary antibody used for each Western blot is indicated at the bottom of each top panel. In the bottom panels, actin was used as a loading control.

Figure S2. Polarity-dependent apical myosin clearing and localization of aPKC and Miranda in ensc, Klp10A-OE and Klp67A-OE NBs

A) Scheme of Myosin clearing from the apical cortex of neuroblast at metaphase/anaphase transition (left). Histogram showing the timing of apical myosin clearing (right) for NBs of the indicated genotypes in control $(30.00 \pm 10.29 \mathrm{~s}, n=18)$, ensc $(34.29 \pm 12.87 \mathrm{~s}, n=21)$, Klp67A-OE $(28.57 \pm 12.92$ s, $n=14)$, Klp10A-OE (26.67 \pm 9.63 s, $n=24)$ NBs. Time 0s is anaphase onset. ns: non-significant. (Wilcoxon test) B) Analysis of aPKC and Miranda localizations in control, Klp10A-OE, Klp67A-OE and in ensc metaphase NBs. Brains of the indicated genotypes were fixed and stained for aPKC (blue), Miranda (green), $\alpha$-tubulin (red) and phospho-histone H3 (Blue). Scale bar: $10 \mu \mathrm{m}$. The number of examined cells (n) is also indicated. None of the conditions used compromise the location of aPKC and Miranda. C) Dot plot showing the NB diameter/GMC diameter ratio ( \pm s.d.) in control $(2.31 \pm 0.20, n=12)$, in $p a v^{B 200} /+(2.32 \pm 0.19, n=20)$, in $s v n^{2180} /+(2.25 \pm-0.11, n=16)$, in Klp10A-OE $(1.93 \pm 0.24$, $n=23)$, in $p a v^{B 200} /+$; Klp10A-OE $(1.73 \pm 0.24, n=21)$, and in $s v n^{2180} /+$; Klp10A-OE $(1.67 \pm 0.27$, $n=16)$ NBs. ns: non-significant, $* *: P<0.01$, ****: $P<0.0001$ (Wilcoxon test). Cell size asymmetry in Klp10A-OE NBs is enhanced by alteration of the CPC and the centralspindlin complex.

Figure S3. Midzone and cortical centralspindlin recruitment in control and colchicinetreated NBs

A) Mean relative cortical/furrow (red) and central spindle (blue) enrichment of Pav-klp-GFP ( \pm s.d.) in a control NB (regarding Figure 4 A). Pav-klp-GFP is rapidly recruited the cortex while its recruitment at the centralspindle is slower. $n=15$. B) GFP-Pav-klp (green and lower panels in monochrome) is strongly recruited to the cell cortex and to the cleavage furrow in Mad2-depleted cells. In the absence of MTs (Mad2 RNAi+colchicine), GFP-Pav-klp is faintly 
787 detected at the basal cortex but is not enriched similarly to conditions where the MT

788 cytoskeleton

$789 \mathrm{n}$ is intact. Scale bar: $10 \mu \mathrm{m}$. Time is s. C) Mean relative GFP-pav-Klp signal ( \pm s.d.) at the

790 cell cortex in $M a d 2$ RNAi with ( $n=9)$ (grey) or without ( $n=7$ ) (dark grey) MTs.

791

792 Figure S4. Analysis of centralspindlin levels at the centralspindle or the cleavage furrow 793 during anaphase

794 A) Dot plot showing the mean relative GFP-Pav-klp signal at the cleavage furrow $180 \mathrm{sec}$ 795 after anaphase onset in control $(0.53 \pm 0.17, n=15)$, ensc $(0.34 \pm 0.09, n=11)$ Klp10A-OE $796(0.36 \pm 0.16, n=12)$, and $\operatorname{sas} 4^{s 2214}(0.48 \pm 0.27, n=10)$, control $(0.32 \pm 0.10, n=7)$, and Klp67A797 OE $(0.18 \pm 0.05, n=11)$ NBs. ns: non-significant, $* * * *: P<0.0001$, (Student test and Wilcoxon 798 test fort Klp67A-OE). B) Dot plot showing the total relative GFP-Pav-klp signal at the 799 midzone $180 \mathrm{sec}$ after anaphase onset in control $(0.22 \pm 0.07, n=15)$, ensc $(0.224 \pm 0.09, n=11)$ 800 Klp10A-OE $(0.46 \pm 0.20, n=12)$, and $\operatorname{sas} 4^{\text {s2214 }}(0.38 \pm 0.13, n=10)$, control $(0.08 \pm 0.04, n=7)$, 801 and Klp67A-OE $(0.12 \pm 0.05, n=11)$ NBs. ns: non-significant, $*: P<0.05, * *: P<0.01, * * * *$ : $802 P<0.0001$, (Student test and Wilcoxon test for Klp67A-OE).

803

804 Figure S5. Analysis of centrosome contribution for cleavage positioning and of 805 peripheral MTs in different backgrounds

806 A) Dot plot showing the mitotic spindle length/NB diameter ratio ( \pm s.d.) in control $(0.85 \pm 0.03, n=12)$ or $\operatorname{sas} 4^{s 2214} \mathrm{NBs}(1.32 \pm 0.17, n=17)$, ****: $P<0.0001$, (Wilcoxon test) B)

808 Dot plot showing the NB diameter/GMC diameter ratio ( \pm s.d.) in control $(2.31 \pm 0.2, n=12)$ or $809 \operatorname{sas}^{\text {s2214 }}$ NBs $(1.94 \pm 0.37, n=19)$. **: $P<0.01$ (Wilcoxon test). C) Selected images of control 810 NBs (top) or NBs in which the basal centrosome has been laser-ablated (bottom). The green 811 triangle shows the basal centrosome before ablation, the red triangle shows the position of the 812 ablated centrosome. The NBs expressed GFP-H2A (to confirm the metaphase stage, anaphase 813 onset and the position of the basal GMC), PH-PLC $\delta$-GFP (to visualize cell contours) and 814 GFP-Aurora A (to target the centrosomes). D) Dot plot showing the NB diameter/GMC 815 diameter ratio ( \pm s.d.) in control $(2.29 \pm 0.21, n=9)$ or basal centrosome-ablated NBs 816 (1.98 $\pm 0.29, n=12)$. ***: $P<0.001$, (Student test). Scale bar: $10 \mu \mathrm{m}$. Time is min:s. E) Selected 817 high-resolution images of control, ensc, Klp67A-OE, and Klp10A-OE NBs. Tubulin is shown 818 in red (and lower panels in monochrome), Myosin is shown in green, and phospho-Histone 819 H3 (Ser10) in blue. The white ellipses show the peripheral MT bundles in the vicinity of the 820 furrow. Scale bar: $10 \mu \mathrm{m}$. F) Histogram showing the number of peripheral astral MTs bundles 
821 detected at the vicinity of the middle cleavage plane in a $\mathrm{Z}$ series projection $(1 \mu \mathrm{m})$ in control

$822(2.3 \pm 0.5, n=9)$, ensc $(1.63 \pm 0.52, n=8)$, Klp67A-OE $(1.7 \pm 0.48, n=10)$ and in Klp10A-OE

$823(0.86 \pm 0.69, n=7)$ NBs. *: $P<0.05, * * *: P<0.001$ (Wilcoxon test).

824

825 Video 1. Asymmetric cell division in a control NB

826 Microtubules (RFP- $\alpha$-tubulin) are displayed in magenta and PH-PLC $\delta$-GFP is displayed

827 in green. Scale bar: $10 \mu \mathrm{m}$. Time is min: $\mathrm{s}$.

828

Video 2. Asymmetric cell division in a Msps-OE NB

830 Microtubules (Msps-RFP) are displayed in magenta and PH-PLC $\delta$-GFP is displayed in 831 green. Scale bar: $10 \mu \mathrm{m}$. Time is min: $\mathrm{s}$.

832

Video 3. Asymmetric cell division in a Klp10A-OE NB

834 Microtubules (RFP- $\alpha$-tubulin) are displayed in red and PH-PLC $\delta$-GFP is displayed in green. Scale bar: $10 \mu \mathrm{m}$. Time is min: s.

836

Video 4. Cortical myosin dynamics during cytokinesis in a control NB

838 Microtubules (mCherry- $\alpha$-tubulin) are displayed in magenta and Sqh-GFP is displayed in green or monochrome (right). Scale bar: $10 \mu \mathrm{m}$. Time is min: $\mathrm{s}$.

Video 5. Cortical myosin dynamics during cytokinesis in an ensc NB

842 Microtubules (mCherry- $\alpha$-tubulin) are displayed in magenta and Sqh-GFP is displayed

843 in green or monochrome (right). Scale bar: $10 \mu \mathrm{m}$. Time is min: s.

845 Video 6. Cortical myosin dynamics during cytokinesis in a Klp10A-OE NB

846 Microtubules (mCherry- $\alpha$-tubulin) are displayed in magenta and Sqh-GFP is displayed in

847 green or monochrome (right). Scale bar: $10 \mu \mathrm{m}$. Time is min: s.

849 Video 7. Pavarotti-klp localization during cytokinesis in a control NB

850 Microtubules (mCherry- $\alpha$-tubulin) are displayed in magenta and GFP-Pav-klp is 851 displayed in green or monochrome (right). Scale bar: $10 \mu \mathrm{m}$. Time is min: $\mathrm{s}$.

852

853 Video 8. Pavarotti-klp localization during cytokinesis in an ensc NB 
854 Microtubules (mCherry- $\alpha$-tubulin) are displayed in magenta and GFP-Pav-klp is

855 displayed in green or monochrome (right). Scale bar: $10 \mu \mathrm{m}$. Time is min: s.

856

857 Video 9. Pavarotti-klp localization during cytokinesis in a Klp10A-OE NB

858 Microtubules (mCherry- $\alpha$-tubulin) are displayed in magenta and GFP-Pav-klp is

859 displayed in green or monochrome (right). Scale bar: $10 \mu \mathrm{m}$. Time is min: $\mathrm{s}$.

860

861 Video 10. Asymmetric cell division in a control NB or after basal centrosome 862 ablation

863 The left panel shows a control NB. The right panel shows a NB in which the basal

864 centrosome was ablated. First and second images show pre and post ablation images

865 respectively before acquisition. Aurora A-GFP, GFP-H2A and PH-PLC $\delta$-GFP are displayed 866 in grey. Scale bar: $10 \mu \mathrm{m}$. Time is min: s.

867

868 Video 11. Fascetto localization during cytokinesis in a control NB

869 Microtubules (mCherry- $\alpha$-tubulin) are displayed in magenta and PH-PLC $\delta$-GFP and Feo-

870 GFP are displayed in green or monochrome (right). Scale bar: $10 \mu \mathrm{m}$. Time is min: s. 


\section{Metaphase}

Cytokinesis

bioRxiv preprintidgi: https://doigrg/t0.1101/2020.09.10.291112, this version posted September 11 , 2020. The copyright holder for this preprint
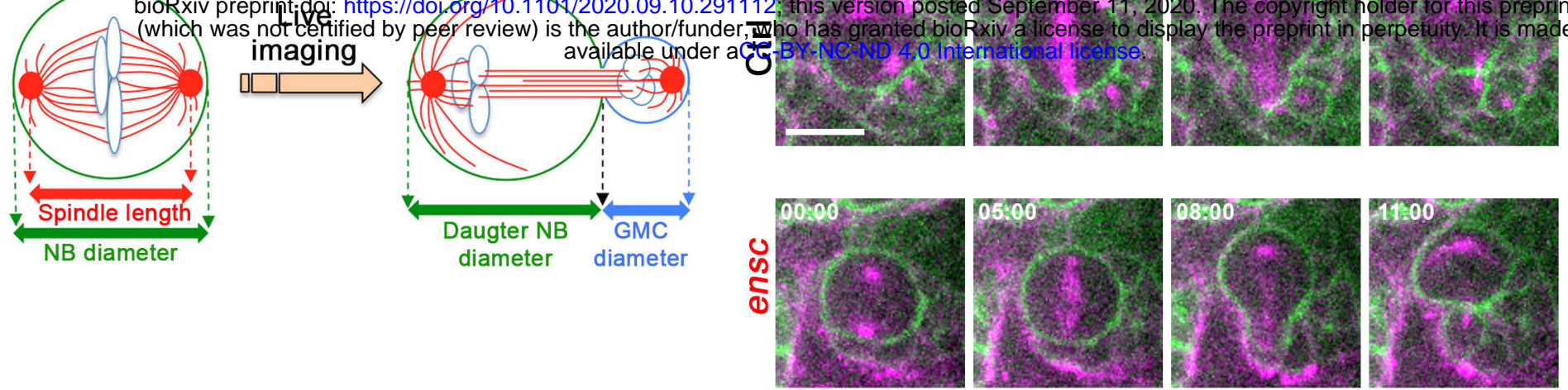

NB diameter
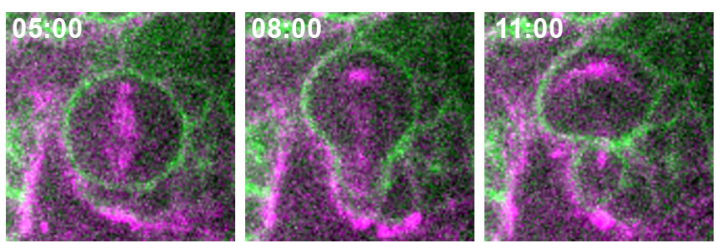

C
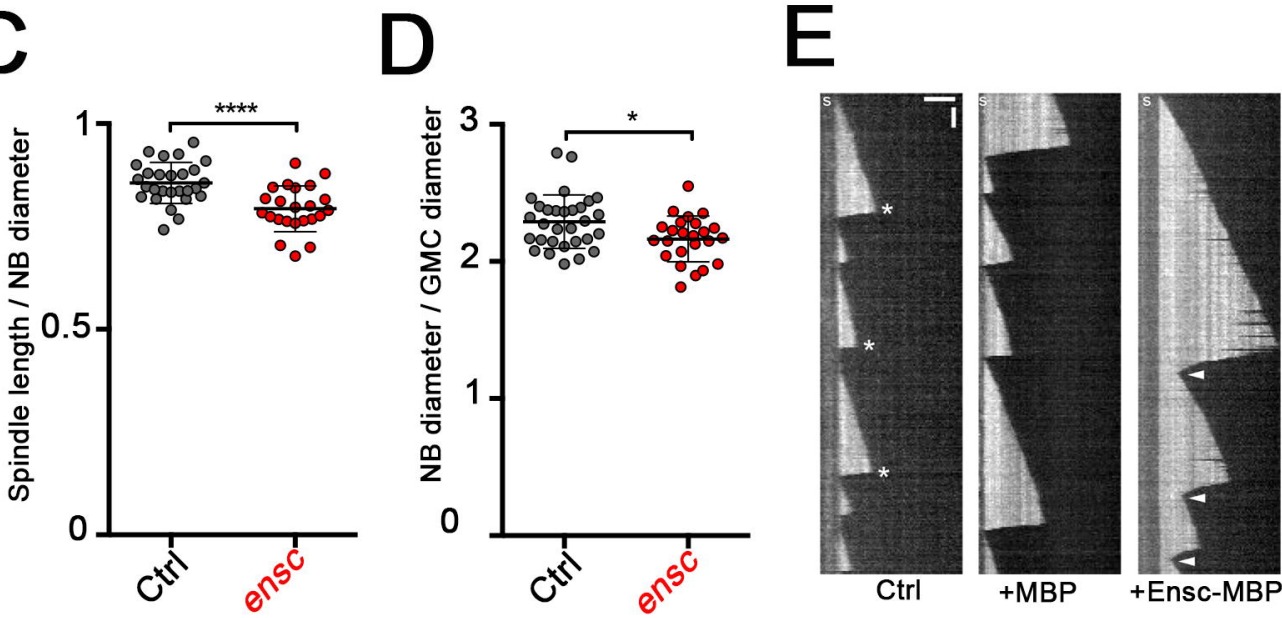

F
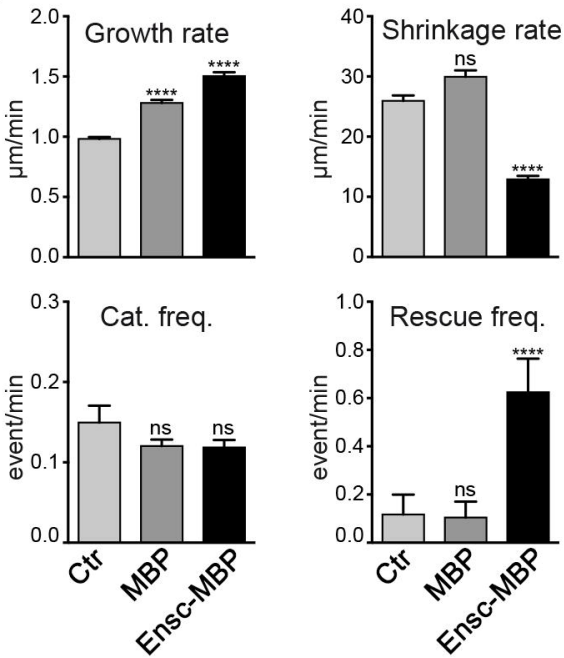

G

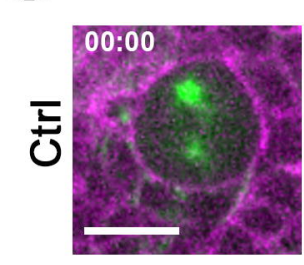

U.

돌

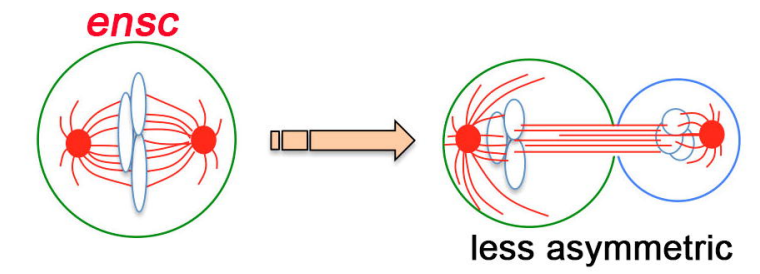

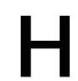
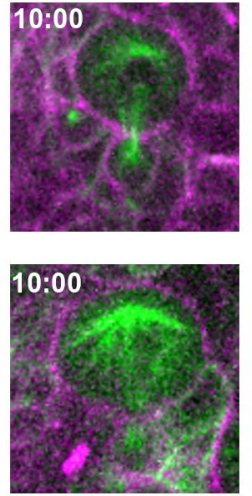

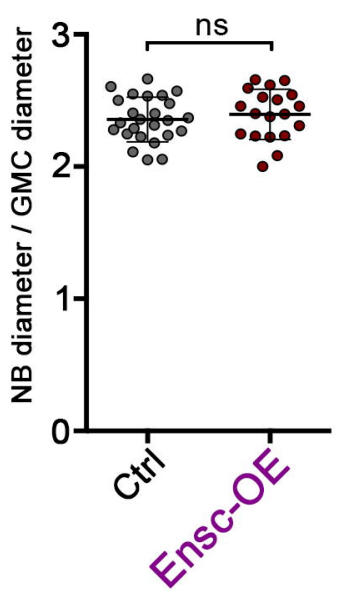



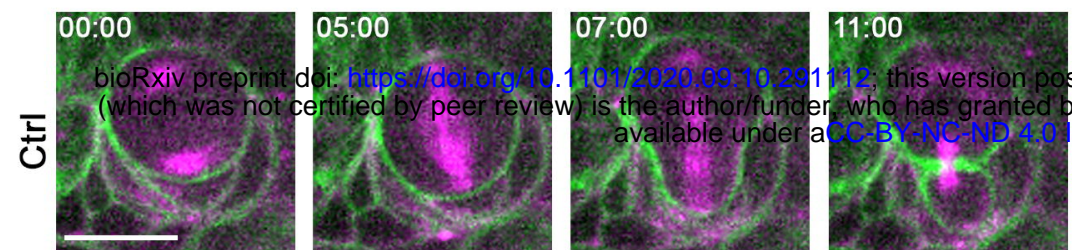

sfed September 11, 2020. The copyright holder for this preprint iaRXIV a license to display the preprint in perpetuity. It is made
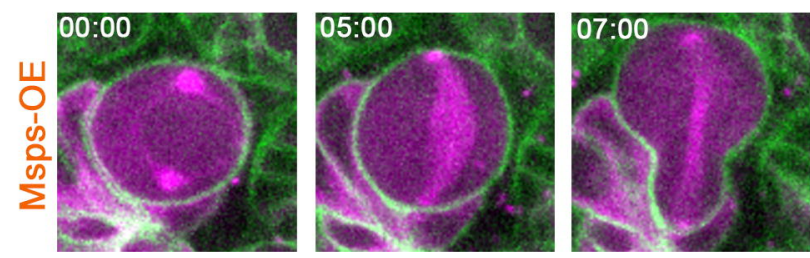

nternational license.
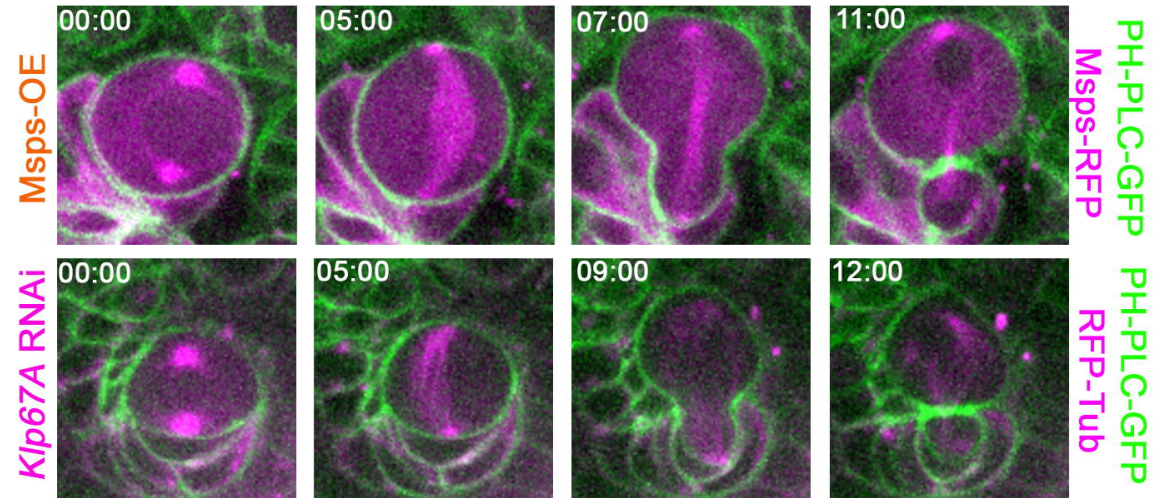

B

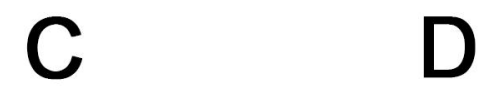

$\mathrm{E}$
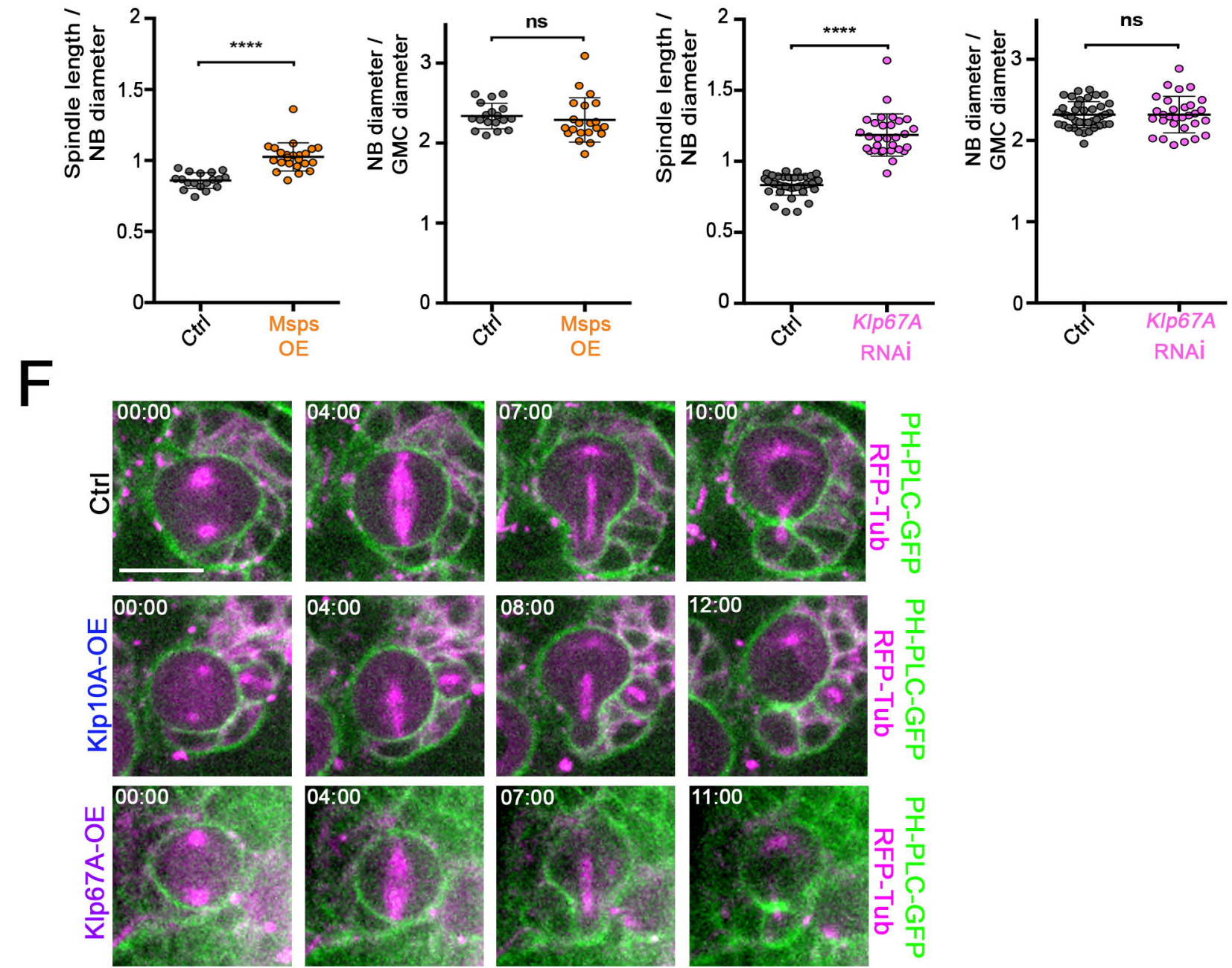

G
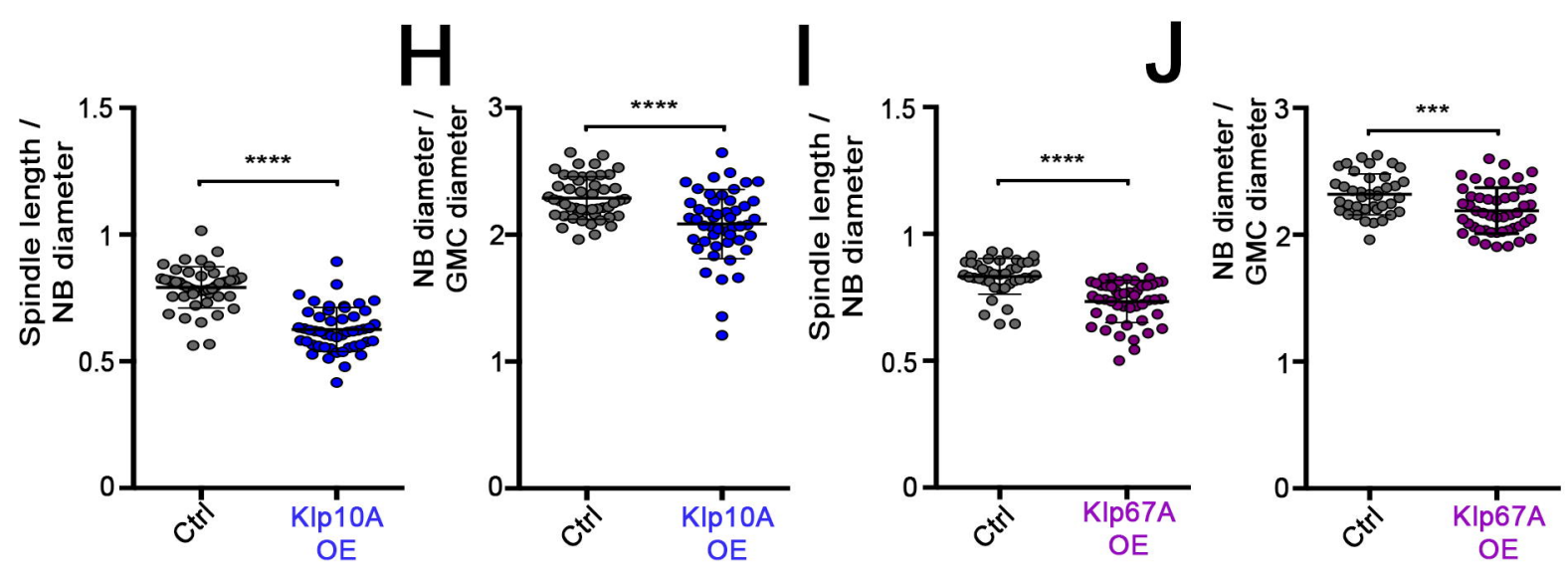

Figure 2 

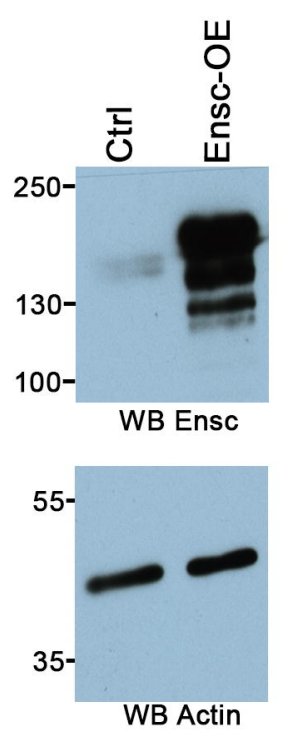
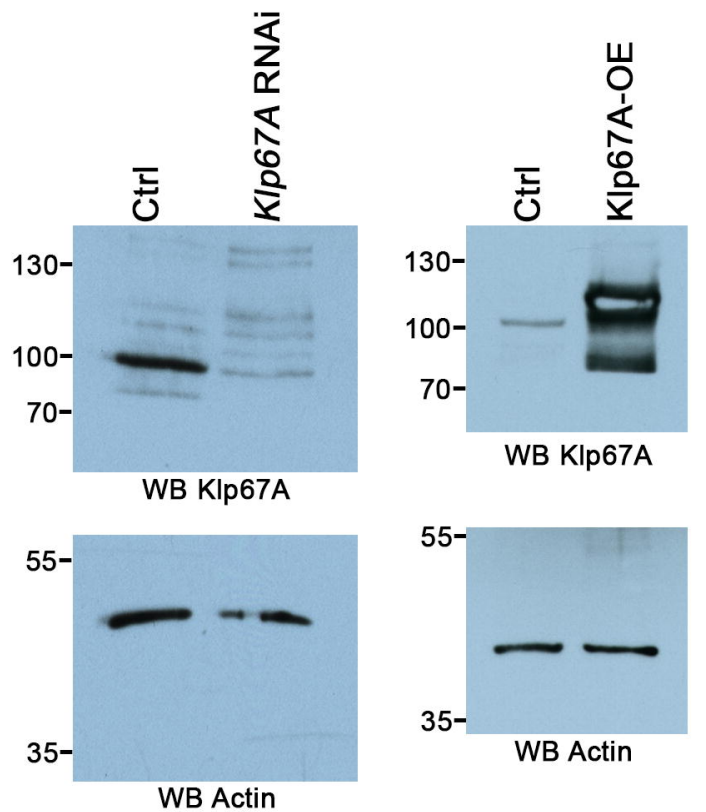
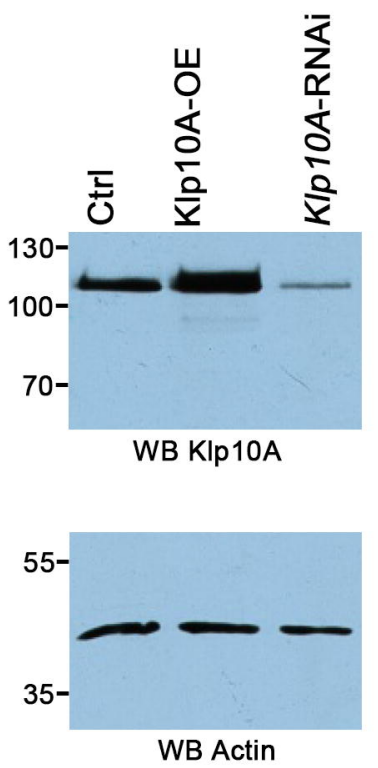

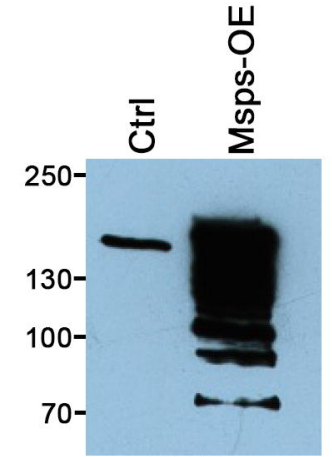

WB Msps

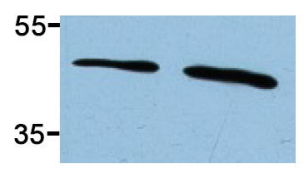

WB Actin

\section{Supplementary Figure S1}


bioRxiv preprint doi: https://doi.org/10.1101/2020.09.10.29111 $\frac{\text { क }}{2}$ this vertion posted September 11, 2020. The copyright holder for this preprint

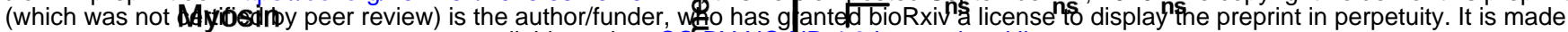

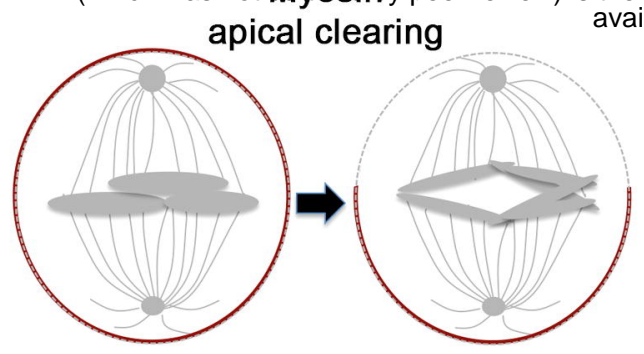
available under aCCS3-NC-ND 4.0 International license.

B

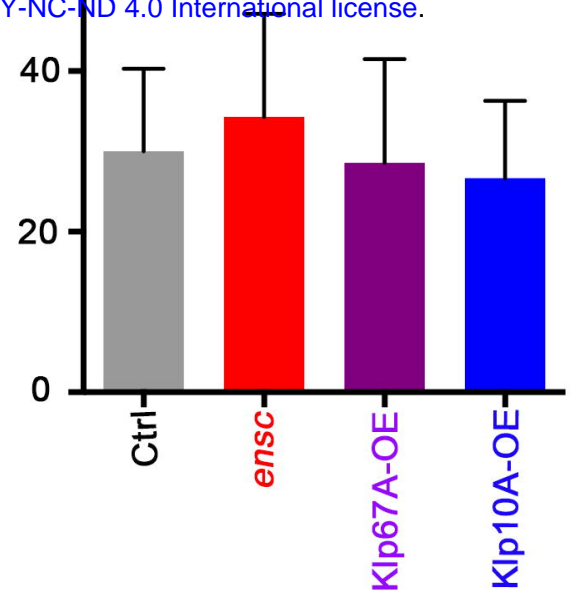

Miranda Tubulin aPKC + PH3
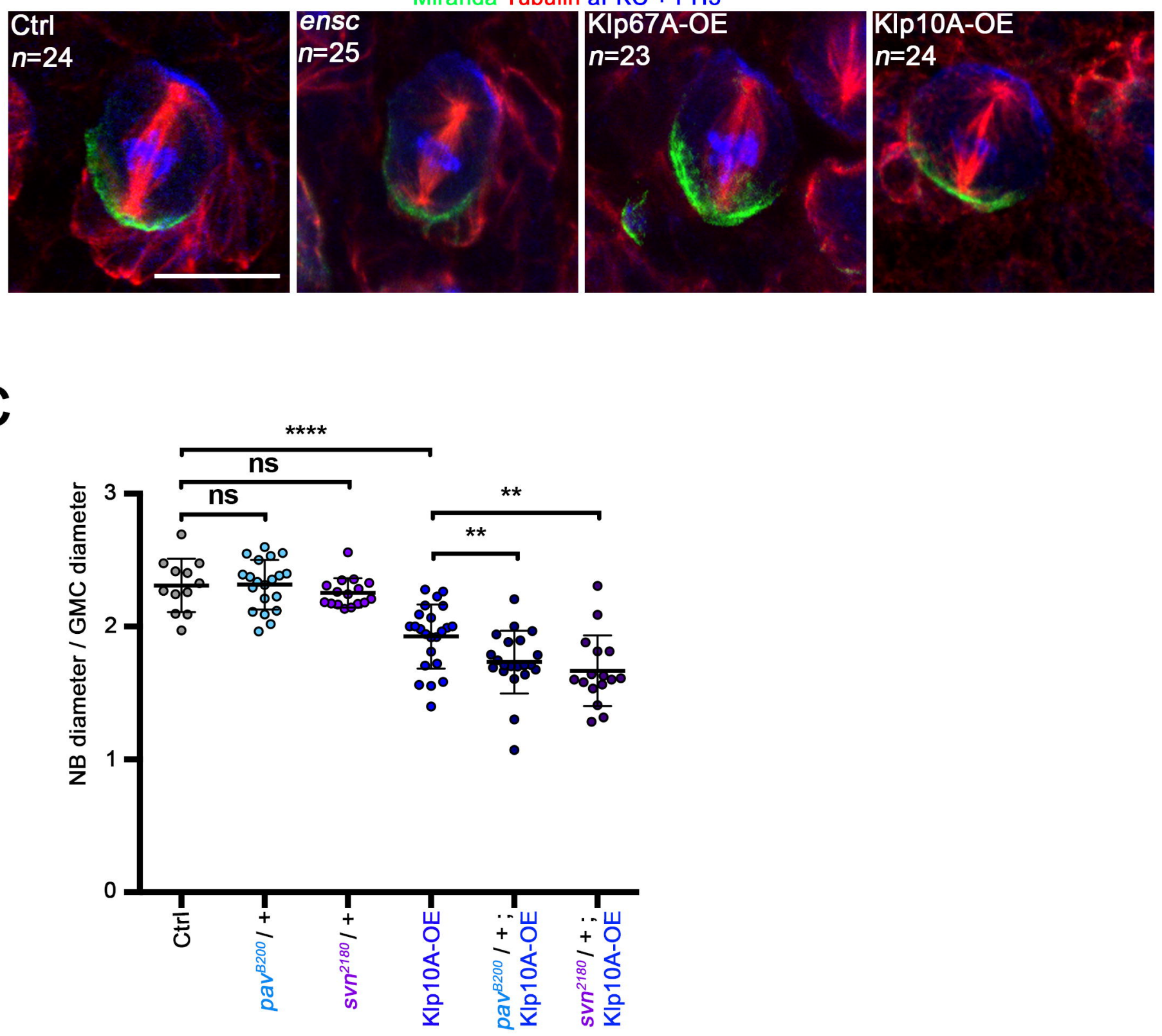
Central spindle

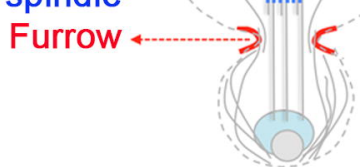

B

\section{Mad2 RNAi}

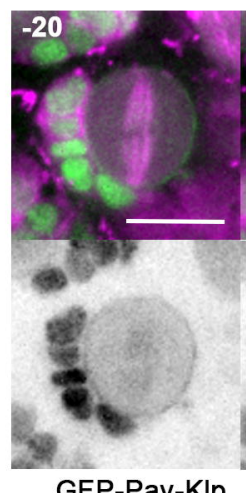

GFP-Pav-KIp

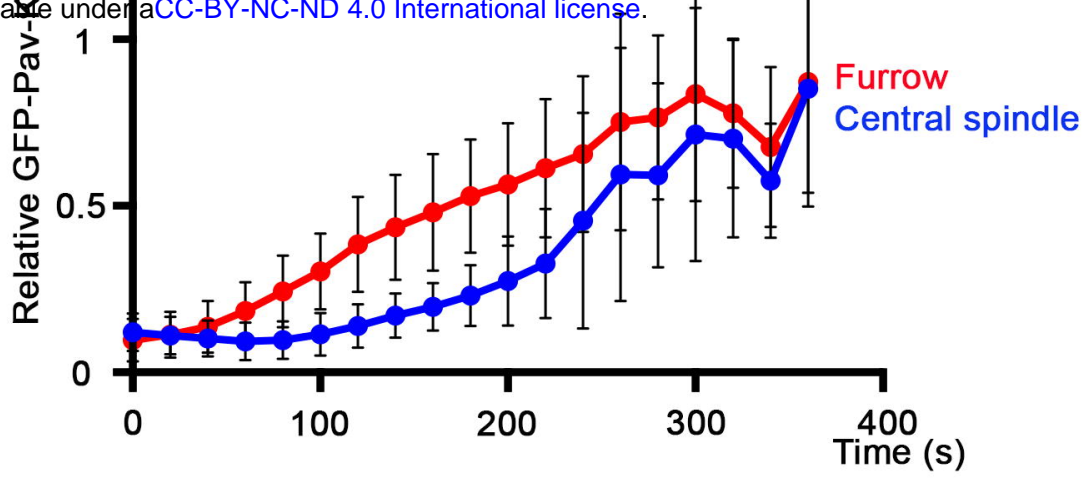

\section{Mad2 RNAi + colchicine}

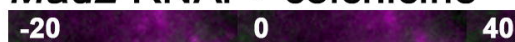

$40 \quad 80$

GFP-Pav-KIp mCherry-Jupiter
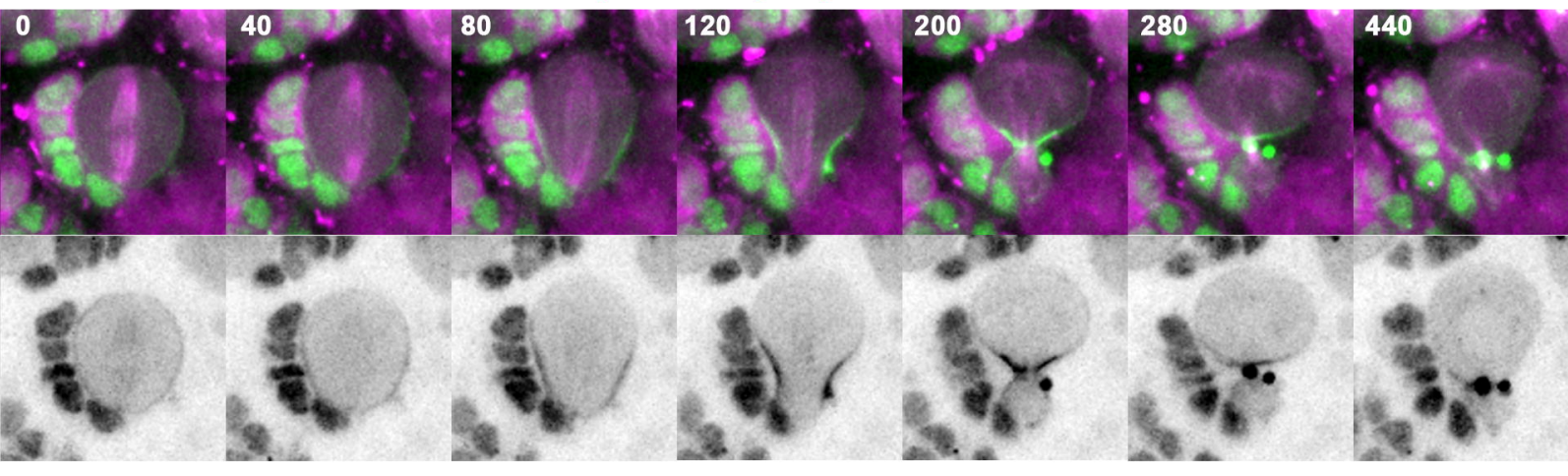

120

200

280

440

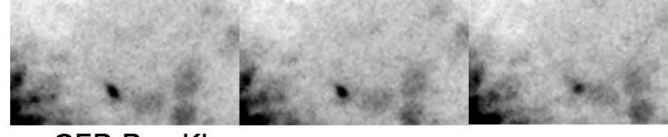

GFP-Pav-KIp

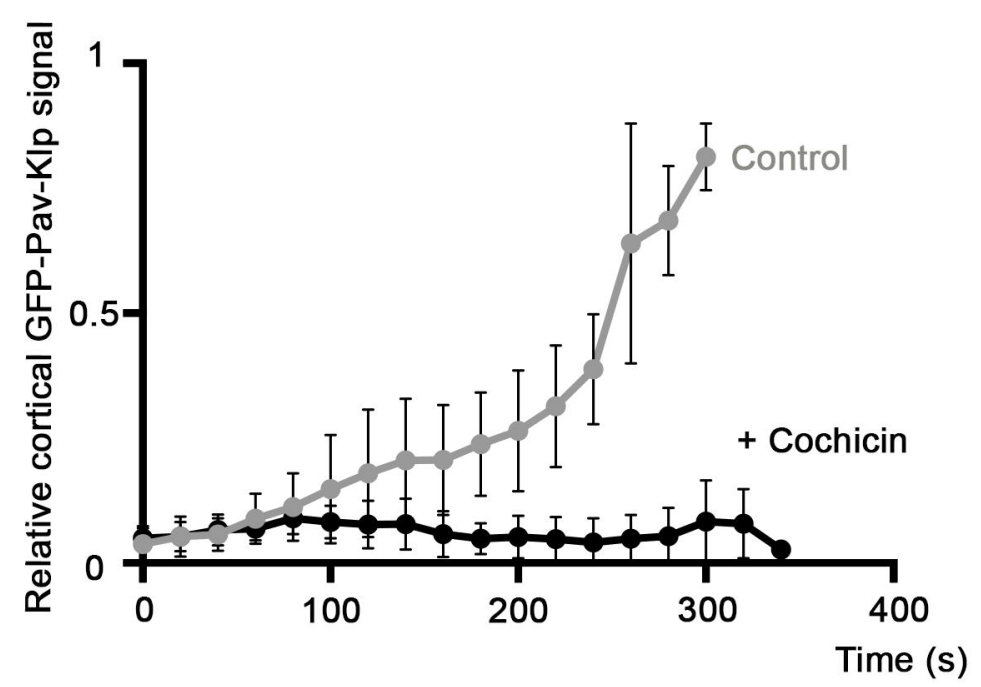


A

bioRxiv preprint doi: https://doi.org/10.1101/2020.09.10.291112; this version posted September 11, 2020. The copyright holder for this preprint (which was not certified by $\mathbf{p a S}$ review) is the author/funder, who has granted bioRxiv a license to display the preprint in perpetuity. It is made

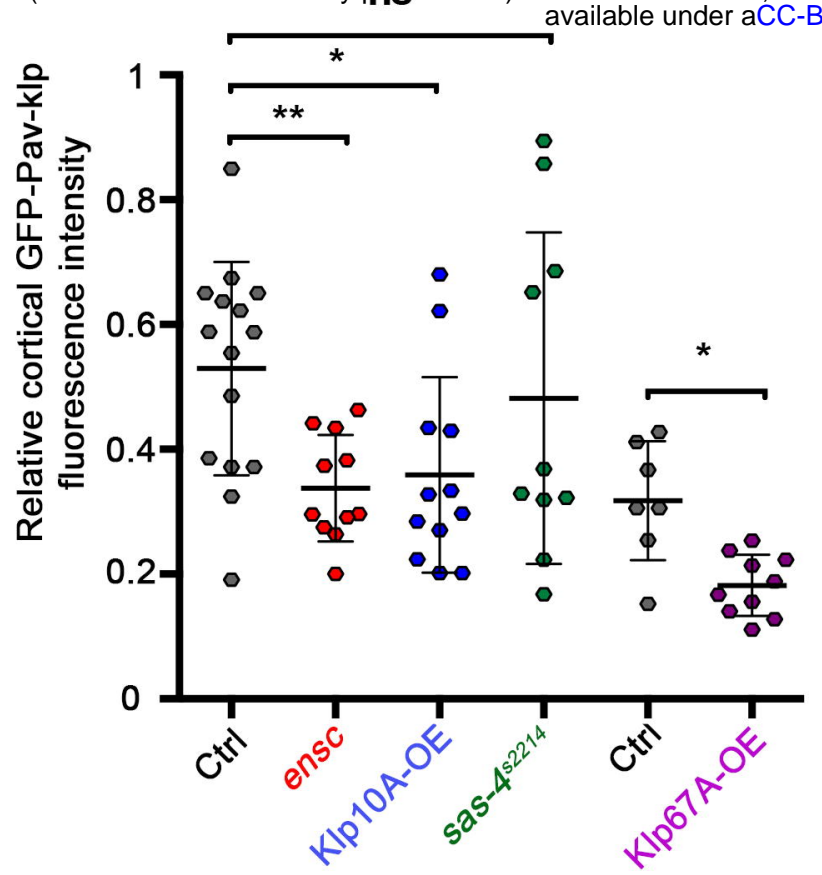

B

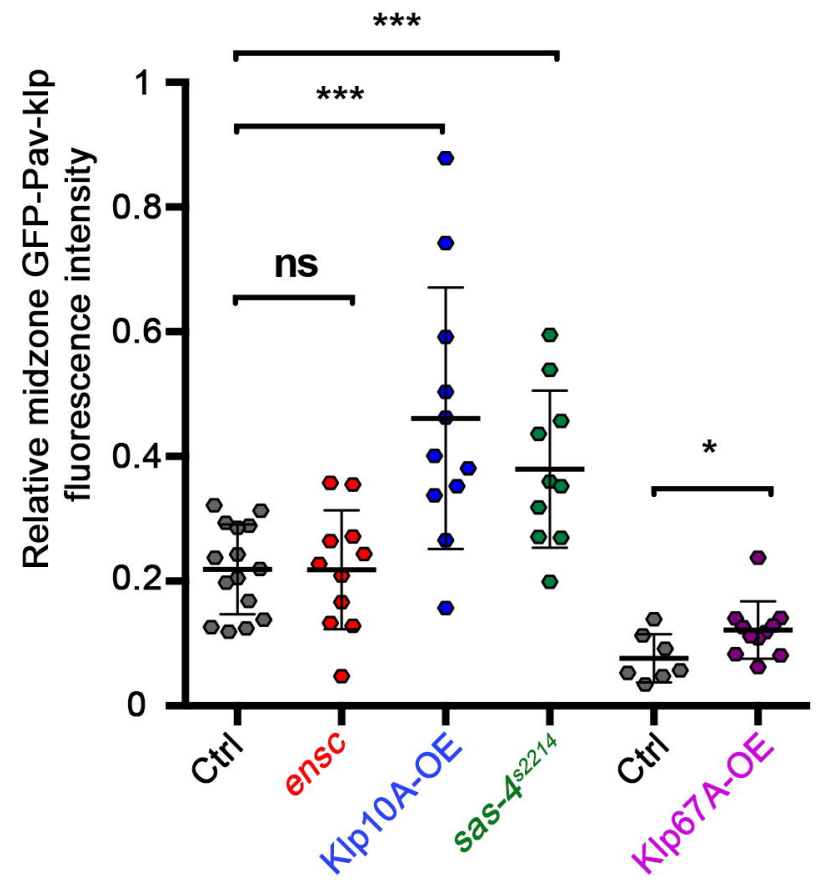


- bioßxiv preprint doi: https://doi.org/10.1101/2020.09.10.291112; *tais version posted September 11, 2020. The copyright holder for this preprint

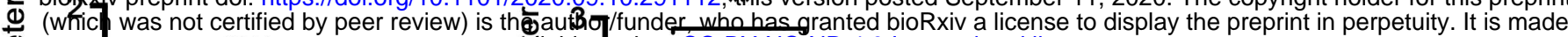

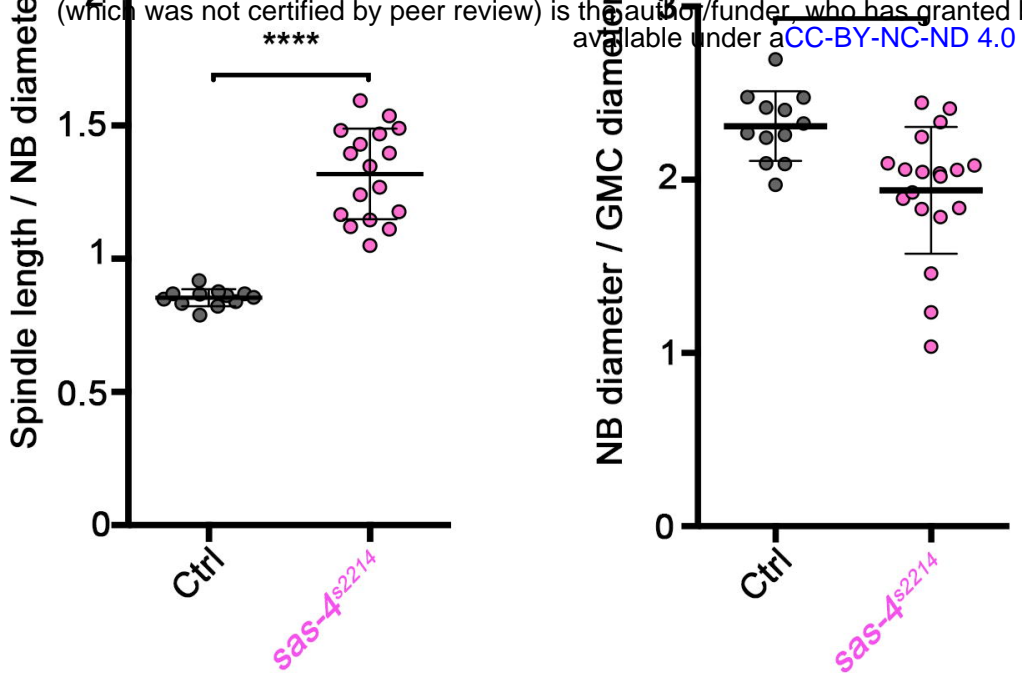

C
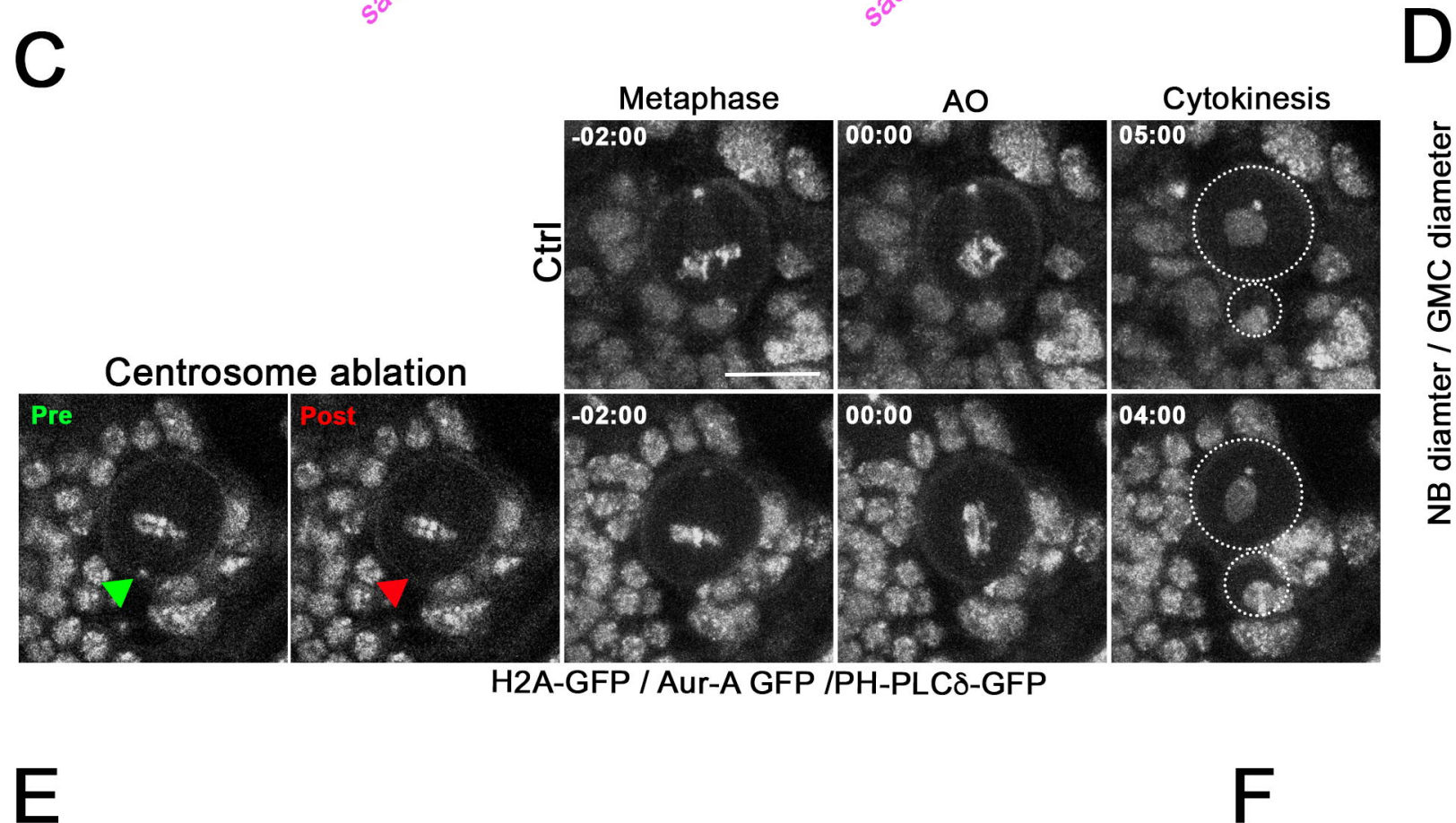

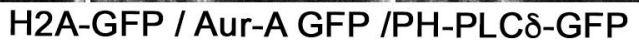
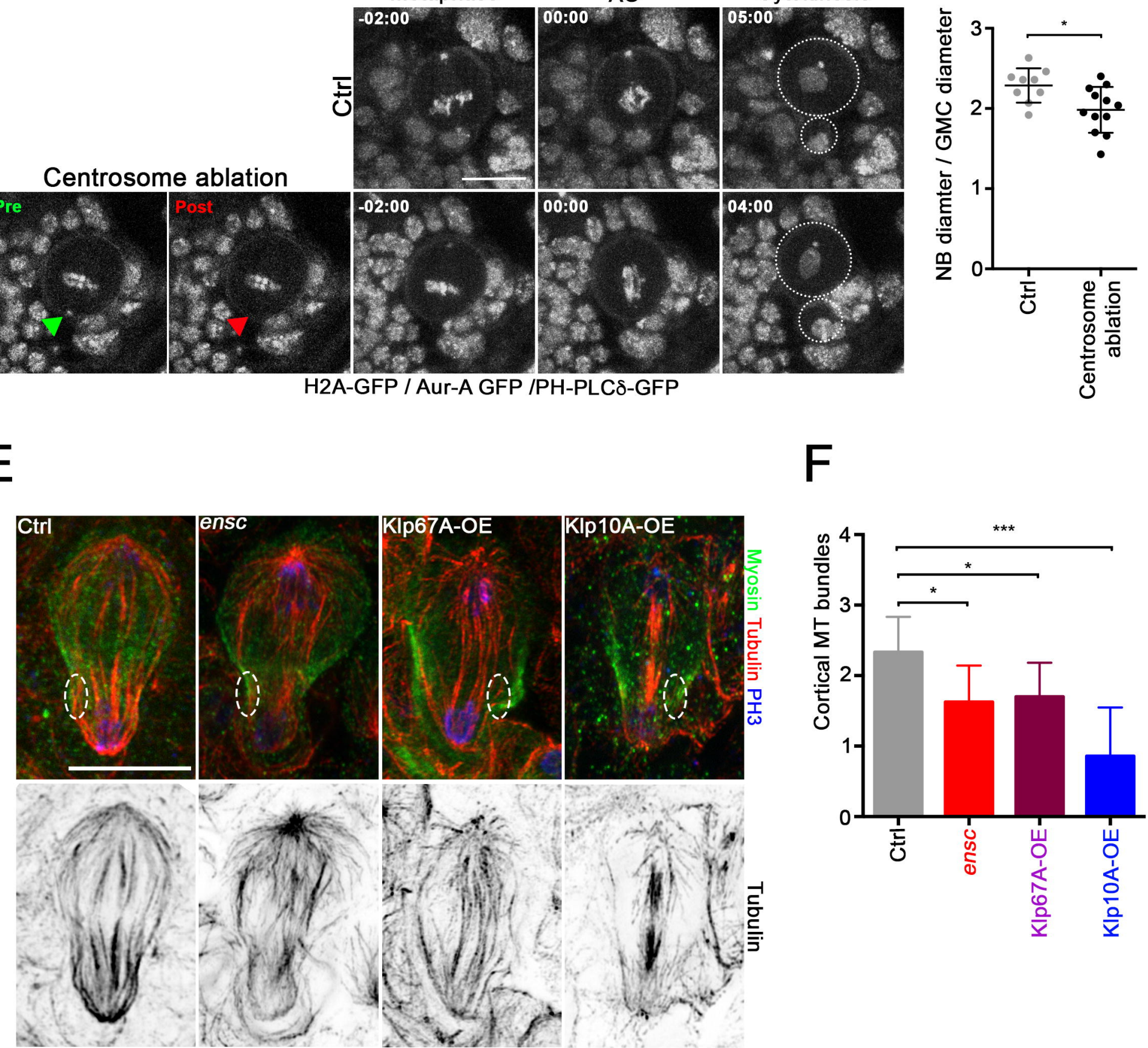